\title{
OVERVIEW OF CORE DIAGNOSTICS FOR TEXTOR
}

\author{
A. J. H. DONNÉ,* M. F. M. DE BOCK, I. G. J. CLASSEN, M. G. VON HELLERMANN, \\ K. JAKUBOWSKA, R. JASPERS, C. J. BARTH, H. J. VAN DER MEIDEN, T. OYEVAAR, \\ M. J. VAN DE POL, and S. K. VARSHNEY FOM-Institute for Plasma Physics Rijnhuizen \\ Association EURATOM-FOM, Trilateral Euregio Cluster, P.O. Box 1207, NL-3430 BE Nieuwegein, The Netherlands
}

G. BERTSCHINGER, W. BIEL, C. BUSCH, K. H. FINKEN, H. R. KOSLOWSKI,

A. KRÄMER-FLECKEN, A. KRETER, Y. LIANG, H. OOSTERBEEK, and O. ZIMMERMANN

Institut für Plasmaphysik, Forschungszentrum Jülich GmbH, EURATOM Association, Trilateral Euregio Cluster

D-52425 Jülich, Germany

G. TELESCA, $\dagger$ G. VERDOOLAEGE $\dagger$ C. W. DOMIER, $\$$ N. C. LUHMANN, Jr., $\$$ E. MAZZUCATO,

T. MUNSAT, ${ }^{\S}$ H. PARK,${ }^{\S}$ M. KANTOR,,$"$ D. KOUPRIENKO,$"$ A. ALEXEEV, ${ }^{\#}$ S. OHDACHI, $* *$

S. KORSHOLM, $\dagger \dagger$ P. WOSKOV, $\dagger \dagger$ H. BINDSLEV, $+\neq$ F. MEO, + \% P. K. MICHELSEN,

S. MICHELSEN, $\$$ S. K. NIELSEN, $+\neq$ E. TSAKADZE, $\$$ and L. SHMAENOK ${ }^{\S \S}$

\author{
$\dagger$ University of Ghent, Belgium \\ $\ddagger$ University of California at Davis, Davis, California \\ $\S$ Princeton Plasma Physics Laboratory, Princeton, New Jersey \\ "Ioffe Physico-Technical Institute, St. Petersburg, Russia \\ ${ }^{\#}$ Multitech, St. Petersburg, Russia \\ **National Institute for Fusion Studies, Toki, Japan \\ $\dagger \dagger$ Massachusetts Institute of Technology, Cambridge, Massachusetts \\ $\$ \uparrow$ Ris $\phi$ National Laboratory, Roskilde, Denmark \\ ${ }_{\S}$ Phystex, Vaals, The Netherlands
}

Received June 4, 2004

Accepted for Publication July 20, 2004

The diagnostic system of TEXTOR comprises about 50 individual diagnostic devices. Since the start of the Trilateral Euregio Cluster collaboration, part of the emphasis in the experimental program has shifted toward the study of physics processes in the plasma core. To aid these studies several new and advanced core diagnostics have been implemented, whereas a number of other core diagnostics have been upgraded to higher resolution, more channels, and better accuracy. In this paper a brief overview is given of the present set of plasma core diagnostics at TEXTOR.

KEYWORDS: TEXTOR, diagnostics, core diagnostics

\section{INTRODUCTION}

From the very beginning of the research on TEXTOR, it has been generally accepted that plasma edge

*E-mail: donne@rijnh.nl physics and plasma-wall interaction cannot be understood without detailed knowledge of the plasma core properties, and a complete set of plasma core diagnostics has been installed on TEXTOR $\left(R / a=1.75 / 0.46 \mathrm{~m}, I_{p} \leq\right.$ $800 \mathrm{kA}$, and $\left.B_{T} \leq 2.9 \mathrm{~T}\right)$. Important contributions to the understanding of the central plasma have been obtained, for example, for the internal magnetic field in a tokamak. In 1996 the three fusion research centers in The Netherlands; Belgium; and North-Rhine Westphalia, Germany, decided to combine their fusion research in the so-called Trilateral Euregio Cluster (TEC) and to concentrate the experimental work on the TEXTOR tokamak. ${ }^{1}$ Although plasma edge physics and plasma-wall interaction still form a substantial part of the TEC physics program, as is evidenced by several papers in this issue ${ }^{2}$ the study of the TEXTOR core plasma has gained in importance. The central research theme for the joint scientific program of TEC is to develop a coherent concept for energy and particle transport and exhaust and their control in fusion reactors. The basic idea behind the research theme is that plasma confinement cannot be understood unless the plasma is considered as a whole, including boundaries. In other words, the control of a burning plasma experiment necessarily encompasses active and interlinked control of the different plasma zones (core, edge, and wall). 
The key elements of the present TEC research program are

1. to clarify the physics mechanisms determining the fluxes in the various confinement zones, with emphasis on the effect of turbulence and selforganized small-scale structures

2. to unravel the interdependencies between the various zones

3. to develop adequate diagnostics and tools to control the fluxes in every zone

4. to develop appropriate theoretical models for this coherent approach.

To aid the aforementioned research program, it was necessary to upgrade various existing core diagnostics and, in parallel, to implement a number of new systems featuring high resolution and good accuracy. An overview of a selection of the new plasma core diagnostics was published a number of years ago. ${ }^{3}$ At that stage many of the diagnostics were still in the design or commissioning phase. Since then, most of the core diagnostics have matured, and therefore, it is in this paper that we present for the first time a complete overview of the core plasma diagnostics for TEXTOR.

Because several of the diagnostics have already been published in literature, it has been decided to keep the descriptions of the individual diagnostics fairly brief. It is our aim to present a concise overview of the core diagnostics for TEXTOR, however, without bothering the reader with an exhaustive description of technical details. Apart from a few exceptional cases, we will also not include references to work of other teams on similar diagnostic systems. For this the reader is referred to the original publications on the various individual TEXTOR diagnostics.

In Secs. II through VI, the core diagnostics for TEXTOR are presented. They are grouped according to the main technique: laser diagnostics, microwave diagnostics, beam-aided diagnostics, passive spectroscopy, and other diagnostics. Although the paper is focused on the present set of core diagnostics, we also will include at certain points flashbacks to successful TEXTOR core diagnostics of the past.

\section{LASER DIAGNOSTICS}

\section{II.A. Interferometry/Polarimetry}

TEXTOR is equipped with a nine-channel interferometer/polarimeter system. The phase change of a far-infrared beam passing through the plasma with respect to a reference beam is proportional to the lineintegrated density (i.e., interferometry). The rotation of the plane of polarization of the beam due to the Faraday effect is proportional to the line-integrated product of the density and the magnetic field component parallel to the beam (i.e., polarimetry). The profiles of density and poloidal magnetic field can be retrieved by numerical Abel inversion of the line-integrated measurements along multiple chords using certain assumptions on the plasma symmetry and taking the Shafranov shift into account.

Figure 1 shows a schematic view of the instrument. The interferometer is a phase-modulated Mach-Zehnder arrangement. It consists of nine probing beams vertically intersecting a poloidal cross section of the plasma. The spacing between the chords is approximately equal, with a better coverage of the low-field side due to the easier accessibility. The reference beam passes outside of the plasma. In addition one horizontal polarimeter channel with a retroreflector on the inside vessel wall equipped with a fast liquid helium-cooled InSb detector is available. The horizontal system is especially useful for measuring fast changes in the vertical plasma position.

The applied light source is a HCN laser with a wavelength of $337 \mu \mathrm{m}$ and a maximum output power of 150 $\mathrm{mW}$. This wavelength has been chosen to fulfill various requirements: On one hand it should be large to give easy measurable phase shifts and Faraday rotation angles; on the other hand it should be short enough to minimize second-order effects like elliptization and beam deflection in regions with strong perpendicular density gradients.

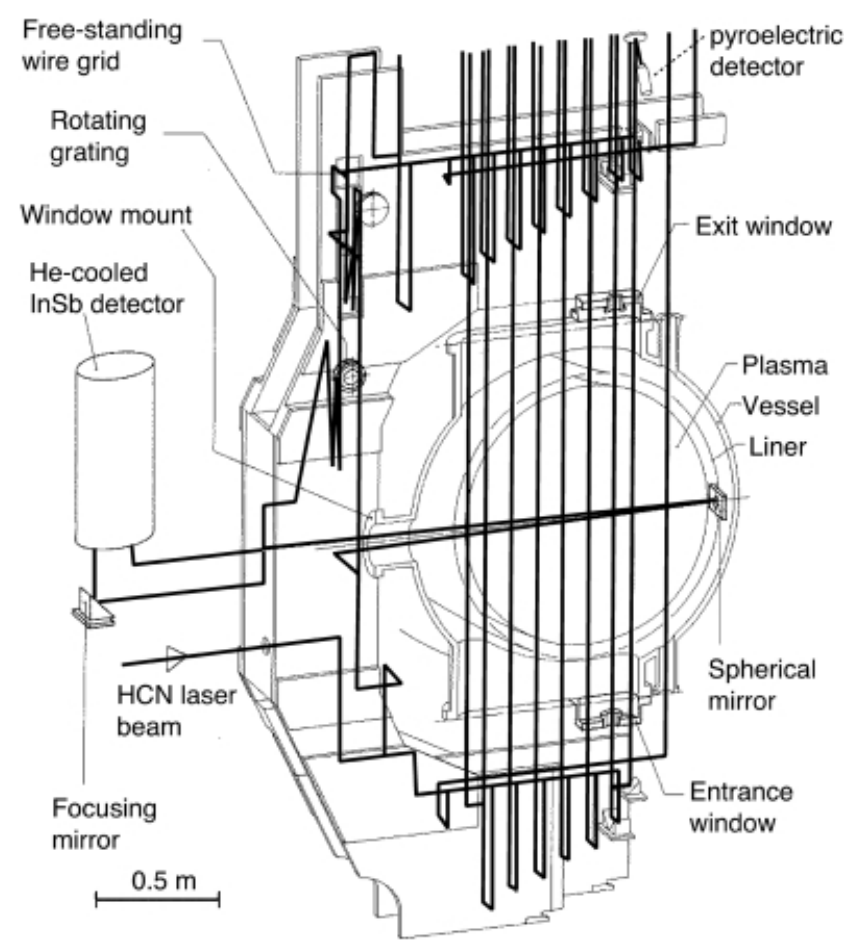

Fig. 1. Schematic drawing of the nine-channel interferometer/ polarimeter on TEXTOR. The line-integrated electron density and the Faraday rotation angles are measured along one horizontal and nine vertical lines of sight. 
Crystal quartz beam splitters divide the HCN laser beam into a reference beam and the various probing beams. All optical components are mounted in a rigid, freestanding, C-shaped frame, which embraces the vacuum vessel. This frame rests on the lower transformer yoke and is vibrationally insulated from all tokamak components.

The basic design of the HCN interferometer follows the guidelines outlined in Ref. 4 but has been extended to enable the simultaneous measurement of the Faraday rotation in order to determine the poloidal distribution of the plasma current density. ${ }^{5}$ The optical arrangement of a probing beam is shown in Fig. 2. The polarization of the beam entering the plasma is aligned perpendicular to the toroidal magnetic field of the tokamak. On exit it is slightly rotated (a few degrees) because of the Faraday effect. A wire grid polarizer combines the probing beam with the reference beam, which is frequency shifted by $10 \mathrm{kHz}$ using a rotating grating. The $\lambda / 2$ plate in front of the beam combiner serves to align the polarization in such a way that the Faraday rotation signal vanishes in the ab-

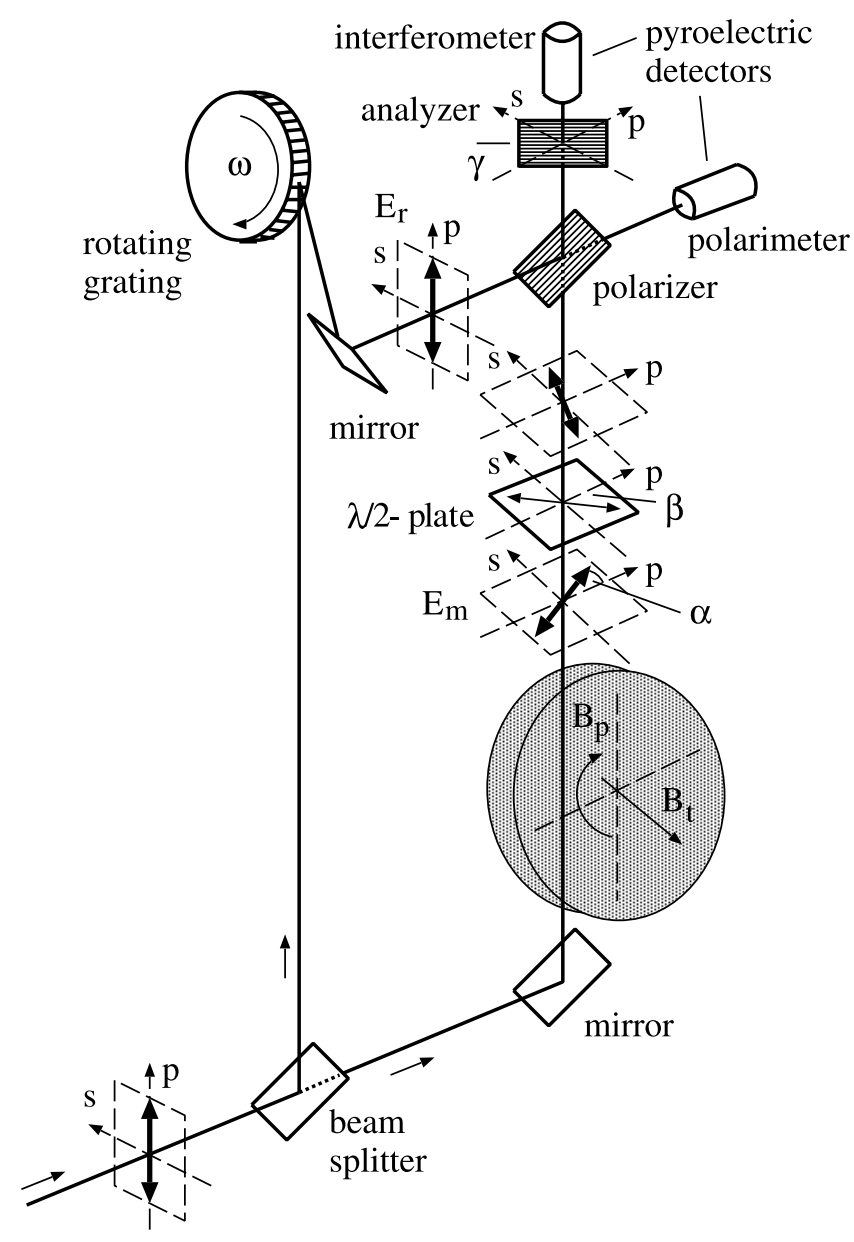

Fig. 2. Optical setup of one probing beam of the combined interferometer/polarimeter. The polarization of the waves is indicated by bold double-headed arrows. sence of a plasma. Rotating this plate by well-known angles allows easy calibration of the rotation angles. The interferometer and polarimeter signals are detected using pyroelectric crystals.

The interferometer signal is determined by a phase comparison of the beat signal from a probing beam with the reference beam. A multifringe real-time phase comparator has been developed ${ }^{6}$ to allow for plasma density and position control. In addition a digital phase measurement with time resolution up to $20 \mathrm{kHz}$ is implemented, too. The total measured phase shifts amount several times $2 \pi$ (a phase shift of $2 \pi$, traditionally called "one fringe," corresponds to a line-integrated electron density of $6.624 \times$ $10^{18} \mathrm{~m}^{-2}$ ), and a noise level as low as $0.01 \pi$ has been achieved at sufficiently high laser output power. The polarimeter signal is obtained by measuring the amplitude with a lock-in amplifier using the interferometer signal of the same probing beam as frequency reference. An absolute resolution of $0.1 \mathrm{deg}$ for the Faraday rotation measurement can be achieved.

A more detailed description of the instrument and the methods applied for data analysis can be found elsewhere. ${ }^{7}$

\section{II.B. High-Resolution Multipoint TS}

In March 2000, a double-pulse, multipoint Thomsonscattering (TS) system was installed to measure the electron temperature and density profile at 120 spatial points along a full vertical chord through the plasma. ${ }^{8}$ The principle of the diagnostic is that the photons in the laser beam are scattered by the electrons in the plasma. The number of scattered photons from any volume in the plasma is proportional to the local electron density in that volume. Because of the thermal motion of the electrons in the plasma, the scattering spectrum is Doppler broadened: Its width is proportional to the square root of the local electron temperature.

The ruby laser $(\lambda=694.3 \mathrm{~nm})$ was mostly operated in a mode with two, 10-12 J, laser pulses, with a time separation of 200 to $500 \mu \mathrm{s}$. The laser beam enters and exits the plasma through vacuum tubes of $\sim 3$-m length, with baffles to reduce stray light from the windows. The laser chord is imaged by means of a six-element lens onto a fiber-optic dissector. Fibers guide the scattered light to a Littrow spectrometer, which is equipped with two separate intensified charge-coupled-device (CCD) cameras to record the two laser pulses. The resemblance of the detector to a television (TV) camera has led to the name "TV Thomson scattering" (TV TS).

The double-pulse TS diagnostic has become a routine instrument and has given input to many physics programs. Electron temperature and density profiles have been measured in a large range of different experiments. High-density radiatively improved (RI) mode profiles turned out to be relatively broad and smooth. Very clear magnetohydrodynamic (MHD) islands have been seen with a density peaking inside the islands, confirming 
earlier work with a prototype four-channel pulsed radar reflectometer. ${ }^{9}$

Although the double-pulse TS system yields electron temperature and density profiles with unprecedented spatial resolution, it has a drawback that it can give at most only two snapshots during a plasma discharge. For this reason the double-pulse system has recently been upgraded to a multipulse TV TS system. ${ }^{10}$ The goal of the new system is to measure with the same high spatial resolution as the double-pulse system on a timescale of $\leq 10 \mathrm{kHz}$ during three bursts of 5 to $10 \mathrm{~ms}$. The system consist of three main parts: (a) an intracavity multipulse laser, (b) a coherent fiber-optic bundle to relay the collected light to the spectrometer, and (c) a Littrow spectrometer equipped with an ultrafast detector. A schematic layout of this system is shown in Fig. 3a (setup of laser and beam line) and Fig. 3b (Littrow spectrometer).

For the new system the Ioffe Physico-Technical Institute in St. Petersburg has developed a so-called intracavity laser. This name indicates that the plasma volume is included in the laser cavity, and hence, the beam travels up and down through the scattering volume. In this way high probing energies can be obtained with only a single ruby rod of $19 \times 200 \mathrm{~mm}^{2}\left(0.03 \% \mathrm{Cr}^{+}\right)$. At a pumping power of $5 \mathrm{MW}$ during $10 \mathrm{~ms}$, a train of up to 50 pulses with $15 \mathrm{~J}$ each can be produced at a maximum frequency of $10 \mathrm{kHz}$. A Pockels' cell is used as an active Q-switch to control the burst of laser pulses. Operation of the laser in a three- or even four-burst mode is in preparation.

After relay and spectral analysis, the scattered light is detected with a complex detector. It consists of a 25-mm Generation III image intensifier with $\sim 50 \%$ quantum efficiency, a stack of three proximity-focused image intensifiers, a tandem lens system, and two fast complementary metal oxide semiconductor (CMOS) cameras for recording. Each camera can sample images with $512 \times$ 384 pixels of $22 \mu \mathrm{m}$ at a frame rate of 10900 frames/s and a 12-bit dynamic range. One camera is used to record the TS light, while the other one is required to sample the plasma light between subsequent laser pulses. This additional recording is needed because of the rather long laser pulse duration of 1.5 to $2.0 \mu \mathrm{s}$, which is 20 times longer than for the previous double-pulse TS system with a laser pulse width of $20 \mathrm{~ns}$. The sensitivity of the CMOS camera of $\sim 50$ photons/count and the light losses of the tandem coupling lens make it necessary to apply additional light amplification. Therefore, a stack of three proximity-focused image intensifiers is used to pile up the photon gain by a factor of 200 . Fast sampling with a

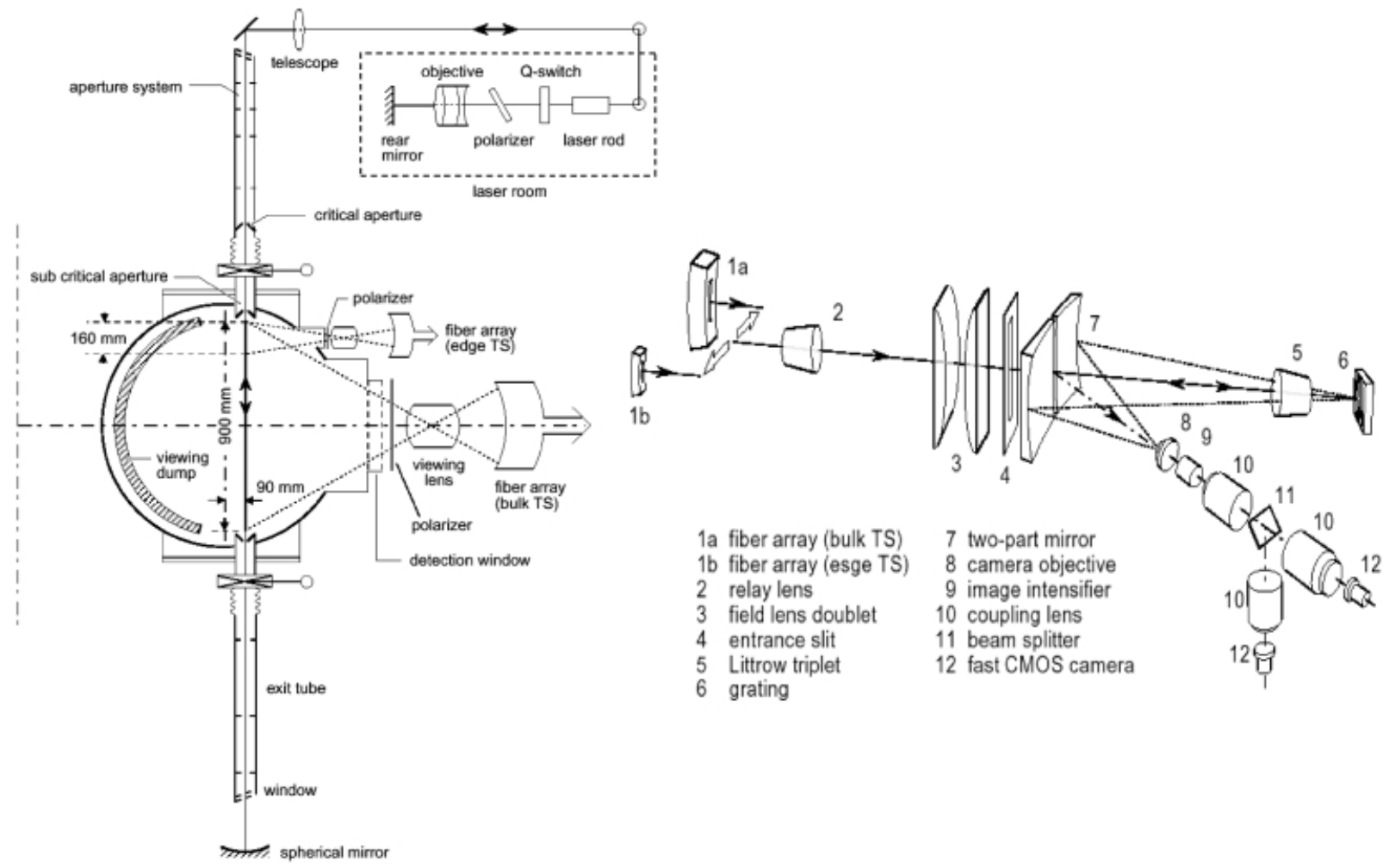

Fig. 3. (a) Schematic overview of the TEXTOR TV TS system with an intracavity ruby laser producing three bursts of $\sim 50$ pulses with $\sim 15 \mathrm{~J} /$ pulse, at a repetition rate of $\sim 10 \mathrm{kHz}$. (b) The collected scattered light is relayed to a Littrow spectrometer giving a 2-D image $(\lambda, z)$. This image is projected onto the high-sensitive cathode of a TV-like detector, capable of recording 12-bit images of $512 \times 384$ pixels with a rate of $10900 \mathrm{frames} / \mathrm{s}$. 
TV-like detector sets special requirements to the imageto-image cross talk. To keep this as small as possible, fast P46 phosphors are used for the output screens of the image intensifiers. The image-to-image cross talk of the CMOS cameras is $\leq 0.02$ when a bias light level is given to the camera (i.e., each image has a $\leq 2 \%$ contribution from the previous image). A fast programmable burst generator (homemade) is used to control the timing of the laser and the detector.

Temperature and density profiles along the full plasma diameter of $900 \mathrm{~mm}$ can be sampled at 120 points with a spatial resolution of $7.5 \mathrm{~mm}$. A separate system for edge observations between $z=340$ and $500 \mathrm{~mm}$ with a resolution of $1.7 \mathrm{~mm}$ has been installed recently. Along the full chord the expected statistical errors on $T_{e}$ and $n_{e}$ for $50 \mathrm{eV} \leq T_{e} \leq 2 \mathrm{keV}$ are $\sim 8$ and $4 \%$, respectively, at $n_{e} \sim$ $2.5 \times 10^{19} \mathrm{~m}^{-3}$. These data were obtained from simulations in which the plasma light is $\sim 25 \%$ of the TS light level, as was extrapolated from experiments with the double-pulse system. Also included was the effect of the different spectral widths due to forward and backward scattering of the downgoing and upgoing beams.

A sequence of ten measured electron temperature and density profiles in TEXTOR for a single burst of laser pulses is shown in Fig. 4. During these very first multipulse TS measurements, the system was not yet calibrated, and moreover, the alignment of the system was not optimized. For this reason one should not interpret the structures in the profiles in Fig. 4 in terms of plasma physics effects, even though many of them are larger than the statistical errors indicated in the previous paragraph.

\section{MICROWAVE DIAGNOSTICS}

TEXTOR has a long history in the field of microwave diagnostics. Electron cyclotron emission (ECE) measurements and reflectometry are still important diagnostics on TEXTOR and are therefore described in the subsequent sections. One microwave diagnostic that has been exploited in the past on TEXTOR, but has been dismantled, is a 2-mm microwave scattering diagnostic. ${ }^{12}$ This system has been used for the measurement of spatially resolved fluctuations. The transmitter and receiver systems both had Cassegrainian antennas that were mounted at the top and bottom of the TEXTOR plasma, respectively.

\section{III.A. ECE Diagnostics}

TEXTOR is equipped with a large number of ECE diagnostics. The ECE systems are based on the fact that
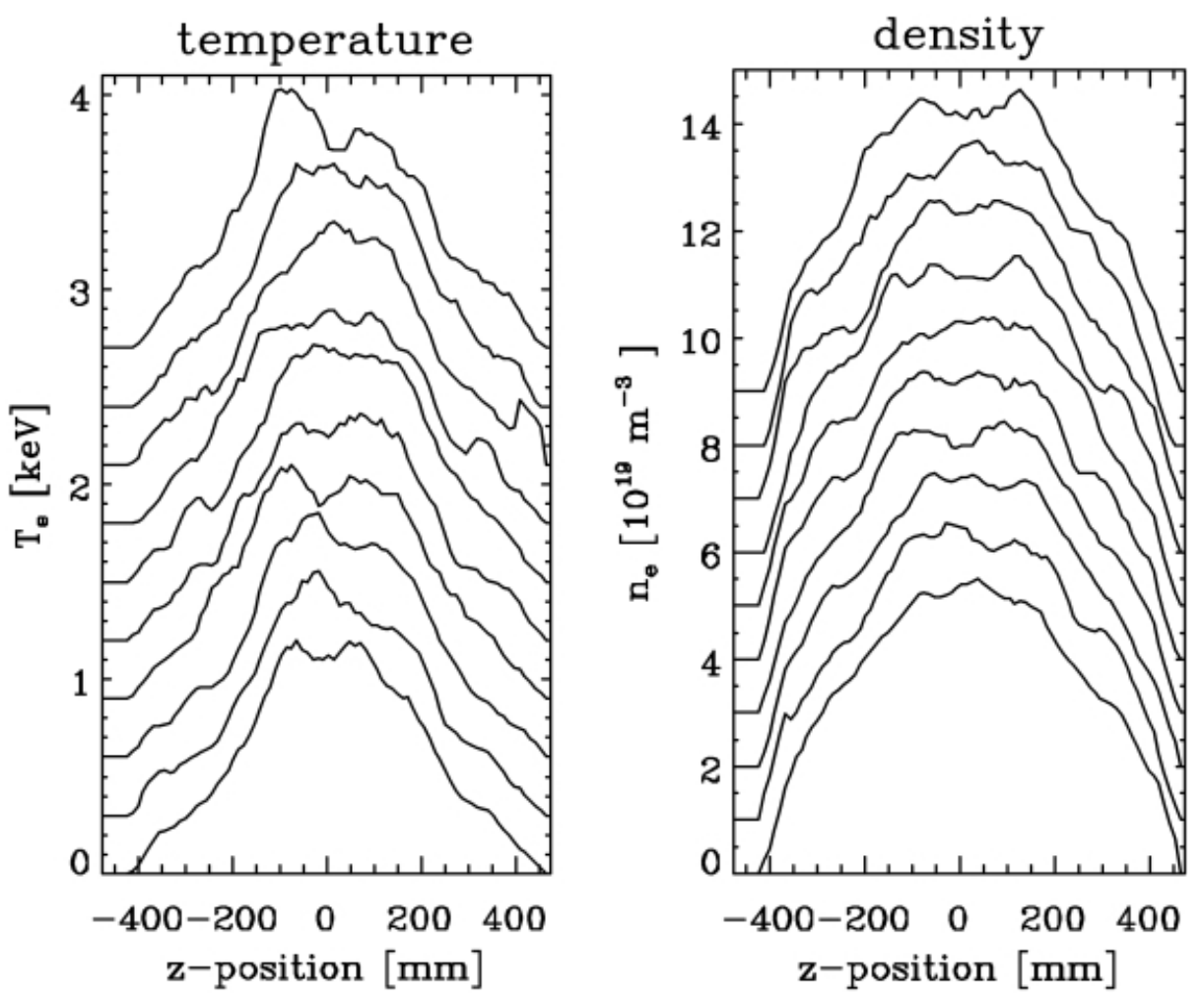

Fig. 4. First ten of $18 T_{e}$ and $n_{e}$ profiles, recorded every $200 \mu \mathrm{s}$, smoothed over four spatial points. The corresponding laser energy ranged from 12 to $8 \mathrm{~J}$. The profiles for $T_{e}$ and $n_{e}$ are plotted above each other with an equidistance of $0.3 \mathrm{keV}$ and $1 \times$ $10^{19} \mathrm{~m}^{-3}$, respectively. Figure taken from Ref. 11 . 
the electrons in the plasma radiate at the electron cyclotron frequency and a number of its higher harmonics. In most cases the plasma is optically thick for the secondharmonic radiation, which implies that it radiates as a blackbody with intensity proportional to the electron temperature. Because the electron cyclotron frequency is a function of the magnetic field, which is proportional to $1 / R$, with $R$ the major radius, it is possible to measure the electron temperature profile by simultaneously measuring the ECE intensity at different frequencies. The various systems at TEXTOR have been developed specifically for some designated purposes:

1. An 11-channel fixed-frequency heterodyne ECE system is used for routine profile measurements.

2. A 4-channel third-harmonic-frequency ECE system is used for measuring temperatures in high-density plasmas for which the second harmonic is in cutoff.

3. Three separate 6-channel heterodyne ECE systems with close channel spacing and channels located near rational $q$ surfaces are used for the study of instabilities.

4. A 16-channel frequency-tuneable ECE system is used for temperature fluctuation measurements.

5. A 128-channel ECE imaging system is used to measure both macroscopic and microscopic temperature perturbations with high time resolution. This system will be described in Sec. III.C.

Routine measurements of the electron temperature profile are done by 11 fixed-frequency single-channel radiometers that diagnose the second-harmonic $X$-mode radiation from the plasma in the frequency range from 105 to $145 \mathrm{GHz}$ (Ref. 13). The use of separate radiometers for each frequency has the advantage of a larger reliability in case of failure of one channel, as compared to systems that use one local oscillator and broadband mixing technology. With a video bandwidth of only $200 \mathrm{MHz}$, the radial resolution is $\Delta r<1 \mathrm{~cm}$. The video amplifiers have a temporal resolution of $100 \mu \mathrm{s}$, which is enough for resolving low-frequency MHD phenomena with $f_{\mathrm{MHD}}<5 \mathrm{kHz}$. The radiometers are connected via several 3 -dB directional couplers to two antennas mounted in the equatorial plane: a long horn antenna at the low-field side and a hog horn antenna at the high-field side. The highfield-side antenna is needed because the channels farthest in the plasma cannot be viewed from the low-field side because of harmonic overlap. For standard TEXTOR operation at $B_{T}=2.25 \mathrm{~T}$, the 11-channel system monitors the region $1.55<R<2.14 \mathrm{~m}$ with a spacing of $0.05 \mathrm{~m}$ between adjacent radiometer channels. The system that is routinely operated during every TEXTOR discharge is absolutely calibrated by means of a modulated blackbody source (at liquid nitrogen and room temperature) with synchronous detection. Temperature profiles are by default calculated on a 10-ms time grid and stored in a common physics database.

Four single radiometers, similar to those described above, at frequencies of $166,170,175$, and $180 \mathrm{GHz}$ are used to monitor the third-harmonic $\mathrm{X}$-mode emission from the plasma. ${ }^{14}$ These radiometers are very valuable to determine the temperature profiles in case the secondharmonic X-mode channel viewing from the low-field side is cutoff, which is the case in high-confinement plasmas, e.g., the RI-mode plasmas. Since the third harmonic is optically thin, information on the local electron density and the reflectivity of the walls is needed to calculate the temperature from the signals. An example for measurements at different harmonics but the same radial position is shown in Fig. 5. The fact that the signal of the secondharmonic channel does not go to zero after the channel is cut off is due to the fact that at the very high densities in these discharges, refraction causes the antenna pattern to become very wide in the vertical direction. Therefore, the antenna can still receive second-harmonic emission from areas below and above the midplane that are not yet cut off.

During the years 2000 and 2001, four secondharmonic channels viewed the plasma via exactly the same antenna as the aforementioned third-harmonic channels. The frequency of the second-harmonic channels was chosen to be exactly two-thirds of that of the thirdharmonic channels. The aim of this combined second-/ third-harmonic system was to study the behavior of suprathermal electrons. ${ }^{15}$ In the absence of suprathermal electrons, the system could also be used to determine the

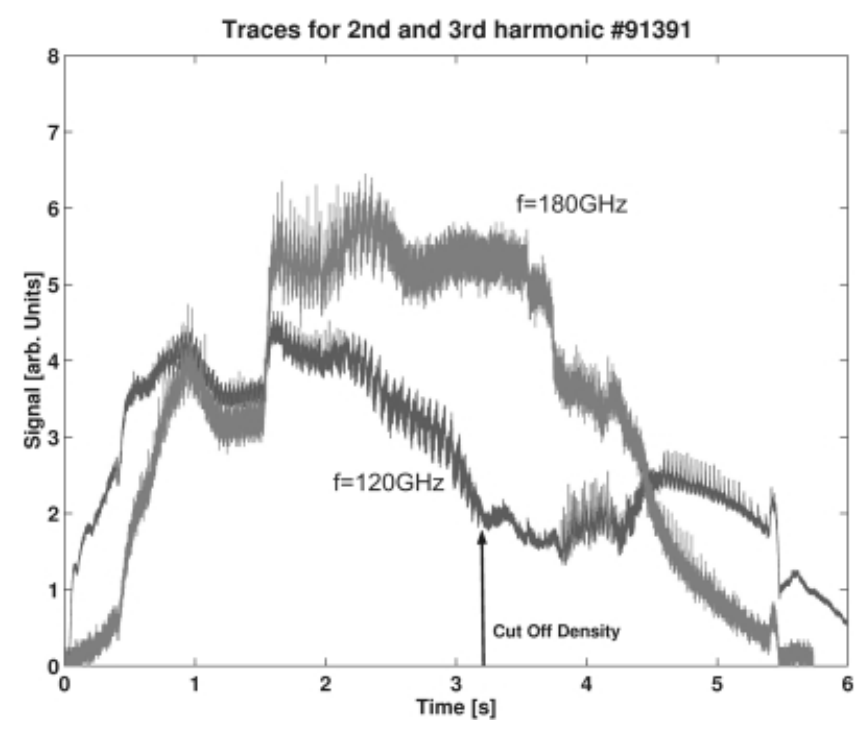

Fig. 5. ECE traces for second- and third-harmonic measurements. Note that the radiation temperature decreases already before the cutoff density is reached at $3.2 \mathrm{~s}$. The difference in the signals is due to the different optical thickness and the reflection of the walls. 
wall reflection coefficient. Because of a shift in scientific interest, the four second-harmonic channels were again removed.

TEXTOR also features a number of high-resolution systems for dedicated research goals: Three 6-channel ECE spectrometers and two frequency-variable radiometers are operated-however not on a routine basis. The spectrometers each work in different frequency bands: 104 to 114,125 to 130 , and 133 to $148 \mathrm{GHz}$ (Ref. 14). Each spectrometer has one local oscillator combined with a broadband mixer of $15 \mathrm{GHz}$. By applying six filters the different channels are realized. The frequency spacing of the channels is 1,2 , and $3 \mathrm{GHz}$, respectively. The channel bandwidth amounts to $400 \mathrm{MHz}$ for all spectrometers, and the temporal resolution can be set to 100,10 , or $1 \mu \mathrm{s}$. All three spectrometers view the plasma via a combination of a hog horn antenna and an elliptical mirror (see Fig. 6). The elliptical mirrors for the three spectrometers are designed to have their focus at the plasma center and in the gradient region on the low- and high-field side, respectively. The elliptical mirrors have a beam waist of $\Delta r \sim 1 \mathrm{~cm}$ and are specially designed for measurements of temperature fluctuations where small observation volumes are needed. With a switch in the waveguide, the antennas can be selected. The two frequency-variable radiometers are also used for temperature-fluctuation measurements. For this reason the frequency can be tuned close to the frequency of one of the spectrometer channels.

With the high temporal resolution, the crash of the sawtooth instability can be followed in detail, and precursor frequencies of up to $12 \mathrm{kHz}$, as observed in neutral beam-heated plasmas, can be easily resolved.

The 11-channel ECE system yields the overall temperature profile but at a rather modest time resolution, whereas the much faster ECE spectrometers cover three

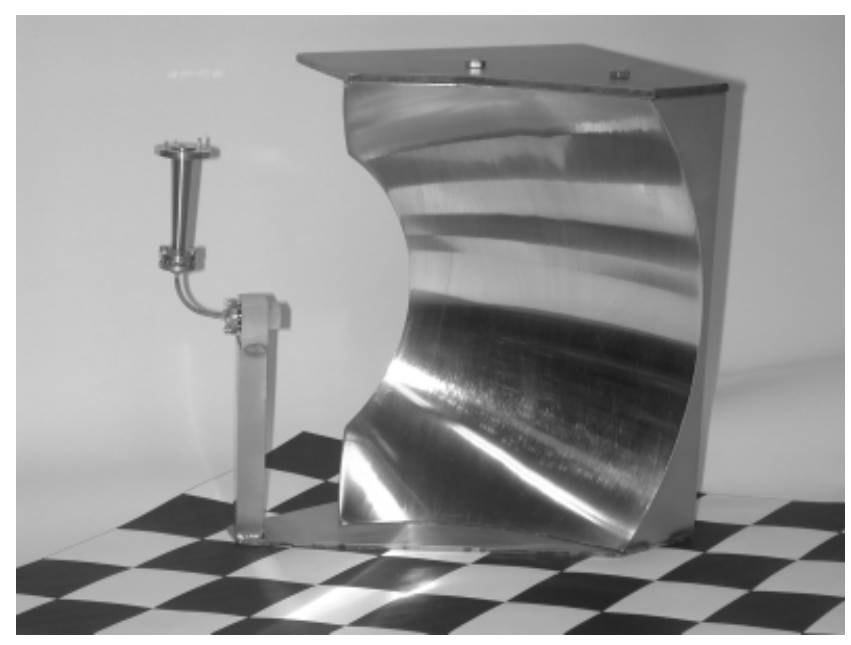

Fig. 6. One elliptical mirror with horn antenna and waveguide. The horn antenna is localized in one of the focal points of the mirror. limited regions inside the plasma. For a standard magnetic field of $B_{T}=2.25 \mathrm{~T}$, the regions observed by the spectrometers are located at those areas where interesting MHD phenomena usually take place. However, for nonstandard plasma conditions it is possible that the observation regions are not at the optimum position. For this reason a 16-channel tunable-frequency ECE radiometer has been recently installed..$^{15}$ The radiometer is of the double-heterodyne type. Four front ends with different local oscillator frequencies convert the input radiation in the frequency range of 98 to $146 \mathrm{GHz}$ to four intermediate frequency (IF) signals with bandwidths of 6 to $18 \mathrm{GHz}$. Each IF signal is down-converted again to four baseband signals. The local oscillators of the second down-conversion step are electronically frequency tunable and are shared by the different front ends. In other words, the frequencies of channels $1,5,9$, and 13 are coupled, as are $2,6,10$, and 14 , etc. The tune range is $3 \mathrm{GHz}$, and the video bandwidth can be set up to $1 \mathrm{MHz}$. The channel frequencies can be dynamically fine-tuned during a TEXTOR discharge to certain regions of the plasma where interesting physics is expected to take place (e.g., MHD islands, transport barriers, etc.). Furthermore, because the distance between the various ECE channels can be changed, it is possible to measure electron temperature fluctuations and correlation lengths by means of correlation ECE.

Finally, by having three major ECE systems at almost 120 deg toroidally from each other (the 11-channel system, the 3- $\times 6$-channel spectrometers, and the 16channel frequency-tunable radiometer), it is well possible to deduce toroidal mode numbers and also to study locked modes. The experimental possibilities are even further enhanced by the fact that TEXTOR also features an 128-channel two-dimensional (2-D) ECE imaging system (see Sec. III.C). Figure 7 shows a 2-D plot of a sawtooth precursor in TEXTOR. The picture has been constructed by using the signals from the various ECE systems at TEXTOR and assuming that the mode rotates, during one rotation period, as a rigid body.

\section{III.B. Reflectometry}

Reflectometry is based on the reflection of microwaves from a critical density in the plasma. For the ordinary mode of polarization, the reflection of the microwaves occurs at the electron plasma frequency. Since each frequency corresponds to a different critical density, it is possible to measure the density profile (or parts of it) by simultaneously reflecting pulses with different frequencies from the plasma. This can be done either as a phase measurement or as a flight-time measurement of short microwave pulses.

\section{III.B.1. Ten-Channel Pulsed Radar Reflectometry}

From 1996 to 2001, a ten-channel pulsed radar reflectometer has been used to monitor the evolution of the 


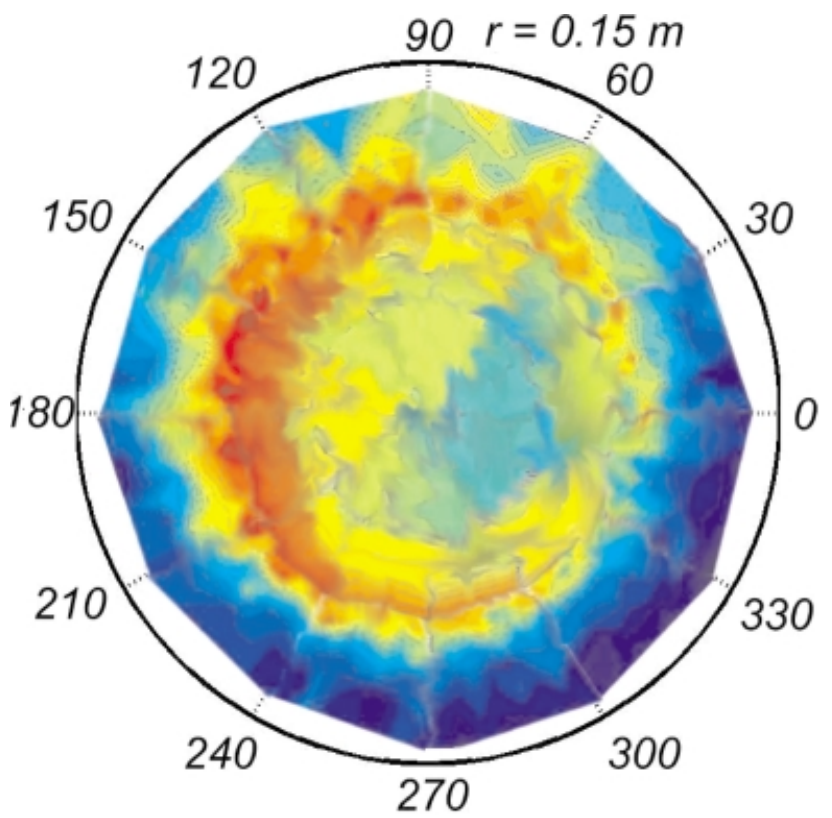

Fig. 7. Two-dimensional reconstruction of a sawtooth precursor in TEXTOR constructed by combining signals from various ECE diagnostics. Figure taken from Ref. 16.

electron density profile as well as coherent and broadband density fluctuations in TEXTOR. The system was based on a direct measurement of the flight time of short $(\sim 1$-ns) microwave pulses that are reflected off the plasma and that contain information on the position of the reflecting density layer. ${ }^{17}$ The system consisted of ten fixed-frequency microwave oscillators launched from the low-field side in the O-mode polarization in the frequency range of 18 to $57 \mathrm{GHz}$ [corresponding to $n_{e}=$ $(0.4$ to 4$) \times 10^{19} \mathrm{~m}^{-3}$ ]. The total pulse repetition rate of $20 \mathrm{MHz}$ could be divided in different ways by a multiplexing technique over the channels, e.g., into a tenchannel profile measurement at $2 \mathrm{MHz}$, a two-channel fluctuation measurement at $10 \mathrm{MHz}$, etc.

The radial accuracy corresponded to $11 \mathrm{~mm}$ when reflecting in vacuum (in plasmas, because of the lower group velocity of the pulse, the accuracy would typically be higher) and could be further improved by averaging subsequent pulses. The diagnostic has already demonstrated to be well suited for detailed studies of macroscopic perturbations of the density profile. It has provided direct evidence of density peaking inside magnetic islands. ${ }^{9,17}$ The density fluctuation level can be determined from the spread in the time-of-flight spectrum of the pulses, and in plasmas exhibiting large $m=2$ MHD modes, it was clearly observed that the high-frequency density fluctuations that are present near the $X$-point of the islands are strongly reduced when the $O$-point of the islands is in front of the antenna. ${ }^{18}$

\section{III.B.2. O-Mode Reflectometry}

At TEXTOR a heterodyne O-mode reflectometry system is installed. It operates at $26<f<37 \mathrm{GHz}$, corresponding to electron densities of 0.84 to $1.7 \times 10^{19} \mathrm{~m}^{-3}$. The plasma path and the reference path are coupled via a

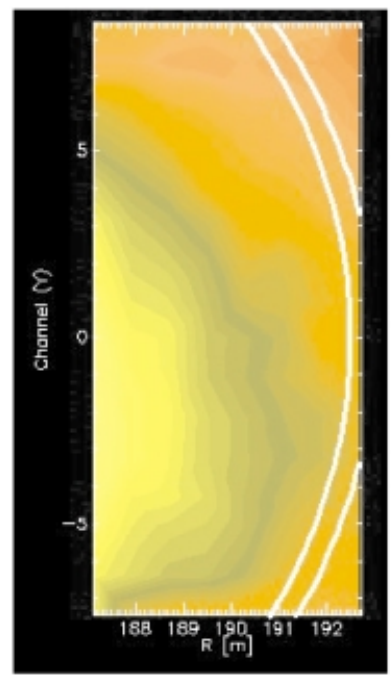

$t=3.058595$

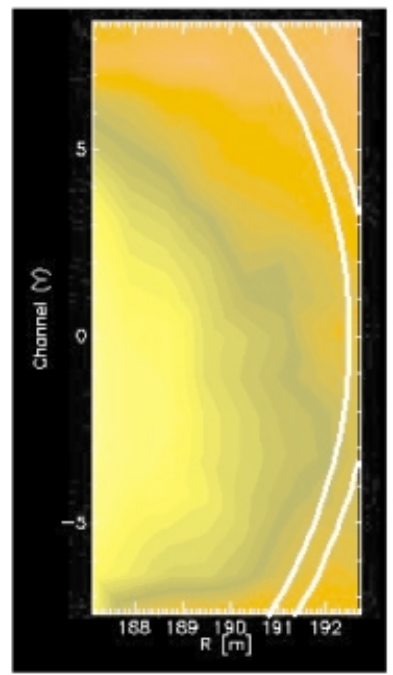

$t=3.058600$

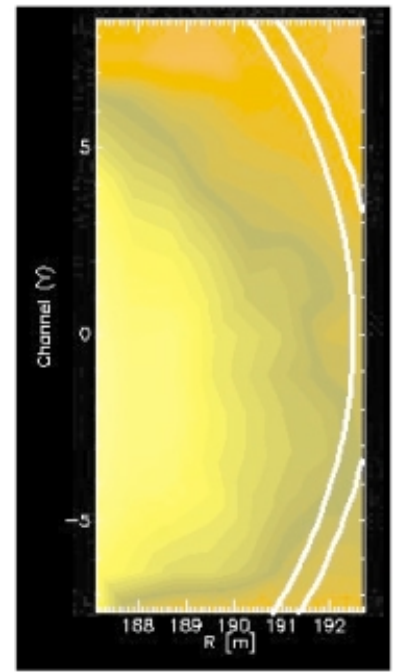

$t=3.058605$

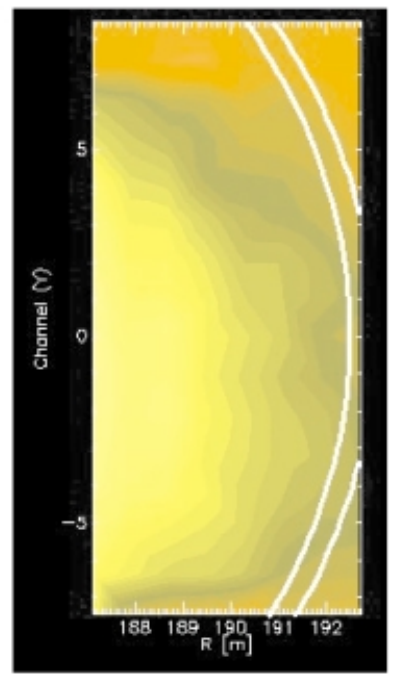

$t=3.058610$

Fig. 11. Sequence of four 2-D temperature perturbation profiles measured by means of ECE imaging during a sawtooth precursor. The $q=1$ radius (determined from the sawtooth inversion) is located between the two white lines. The yellow colors indicate a temperature that is higher than the average value at that position, while the orange colors indicate a temperature that is slightly lower. 
phase locked loop. The frequencies of the two oscillators differ by $20 \mathrm{MHz}$. The system can be coupled via switches to different antenna arrays in the equatorial plane and at the top of the vessel. Each antenna setup consists of five antennas aligned with and focused on the plasma center at $R=1.75 \mathrm{~m}$. The principal setup with an array of three antennas is shown in Fig. 8. Any combination between the antennas in both arrays is possible. The reflected signal is mixed with the reference signal, and the resulting $20-\mathrm{MHz}$ output signal is detected by a quadrature detector for each antenna. The quadrature detector yields a sine and cosine output that is stored by a fast acquisition system with a sampling rate that can be set to 500 $\mathrm{kHz}$ or $1 \mathrm{MHz}$.

The reflectometer is used for the measurement of small-scale structures in the plasma. Also, low $m$-number MHD instabilities can be studied. The different antennas can furthermore be used for cross-correlation reflectometry. Within each array the poloidal angle for the crosscorrelation analysis is in the range 2.5 to $6.5 \mathrm{deg}$, depending on the location of the cutoff surface in the plasma. This makes it possible to determine the poloidal propagation of the small-scale structures along the reflection layer. Taking different antenna combinations into account, one can obtain the correlation length as well as the correlation time.

From the amplitude spectrum of cross-correlation reflectometry and the amplitude spectrum of the anten-

\section{$\underline{\text { TEXTOR }}$}

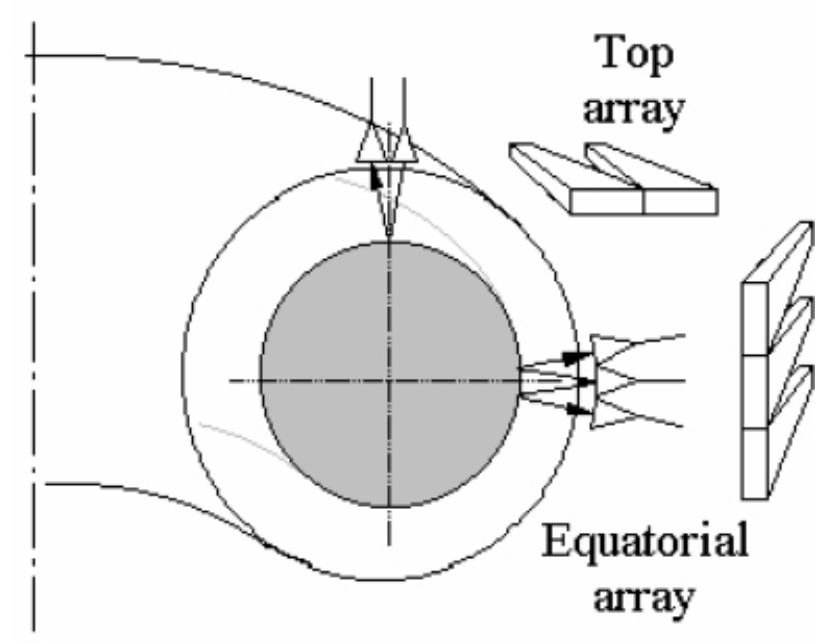

$$
\begin{aligned}
& \text { Polarization: O-mode } \\
& \mathrm{F}=26.0-37.5 \mathrm{GHz} \\
& \mathrm{N}_{\mathrm{e}}=0.84-1.74 \mathrm{E}+13 \mathrm{~cm}^{-3}
\end{aligned}
$$

Fig. 8. The principal setup of the reflectometry at TEXTOR with a reduced antenna array. nas, three different kinds of structures can be recognized (see Fig. 9). First, one usually sees a noncorrelated broadband turbulence. This kind of turbulence is present only in the amplitude spectrum but vanishes in the coherency spectrum. Second, on top of the broadband-turbulence, quasi-coherent structures often show up. These smallscale structures have high $m$ numbers in the range of 40 to 60 at TEXTOR and show up in the spectrum between 50 to $100 \mathrm{kHz}$ depending on the plasma parameters. The plasma sometimes even shows two quasi-coherent modes at different frequencies. Finally, low-frequency structures are often observed $(f<30 \mathrm{kHz})$. The quasicoherent structures dominate the gradient region whereas the low-frequency structures are found close to the plasma edge.

The phase signal of an antenna can also be used to estimate the root-mean-square (rms) fluctuation level as well as the density fluctuation level at the reflection layer. Since the rms level is related to the transport coefficients, reflectometry is an interesting diagnostic for the detection of transport barriers.

\section{III.C. ECE Imaging/Microwave Imaging Reflectometry}

Diagnostic systems for fluctuation measurements in plasmas have, of necessity, evolved from simple onedimensional (1-D) systems to multidimensional systems because of the complexity of turbulence physics of plasmas illustrated by advanced numerical simulations. Using the significant advancements in millimeter-wave imaging technology, ${ }^{19}$ microwave imaging reflectometry ${ }^{20}$ (MIR) and ECE imaging ${ }^{21}$ (ECEI), capable of measuring density and temperature fluctuations, are developed. Both systems require large collection optics for the reflected waves from the "cutoff layer" in the MIR system and vertically (poloidally) extended emissions in the ECEI system. Because both systems operate in the X-mode and in a similar microwave range (the MIR frequency range is $\sim 89 \mathrm{GHz}$, and ECEI ranges from 95 to $130 \mathrm{GHz}$ for TEXTOR), it is feasible to combine the two systems. Reflectometry signals are separated from the ECE radiation by a beam splitter to allow simultaneous temperature and density fluctuation measurements. Both systems utilize state-of-the-art millimeter-wave planar detector arrays positioned at the focal point of the optical system that forms an image of the plasma fluctuations on the detector plane (see Fig. 10) (Ref. 22).

In previous ECEI systems, including the first ECEI system that was used on TEXTOR (Ref. 21), the measurements were essentially $1-D$ in nature in that the detected radiation from each array element was sampled at only a single frequency at a given time. This is in contrast to the conventional wideband ECE radiometer, in which multiple frequency elements are simultaneously detected from a single antenna resulting in a horizontally aligned sampling. A step forward is the 2-D ECE imaging system that has been recently installed on TEXTOR. This system 

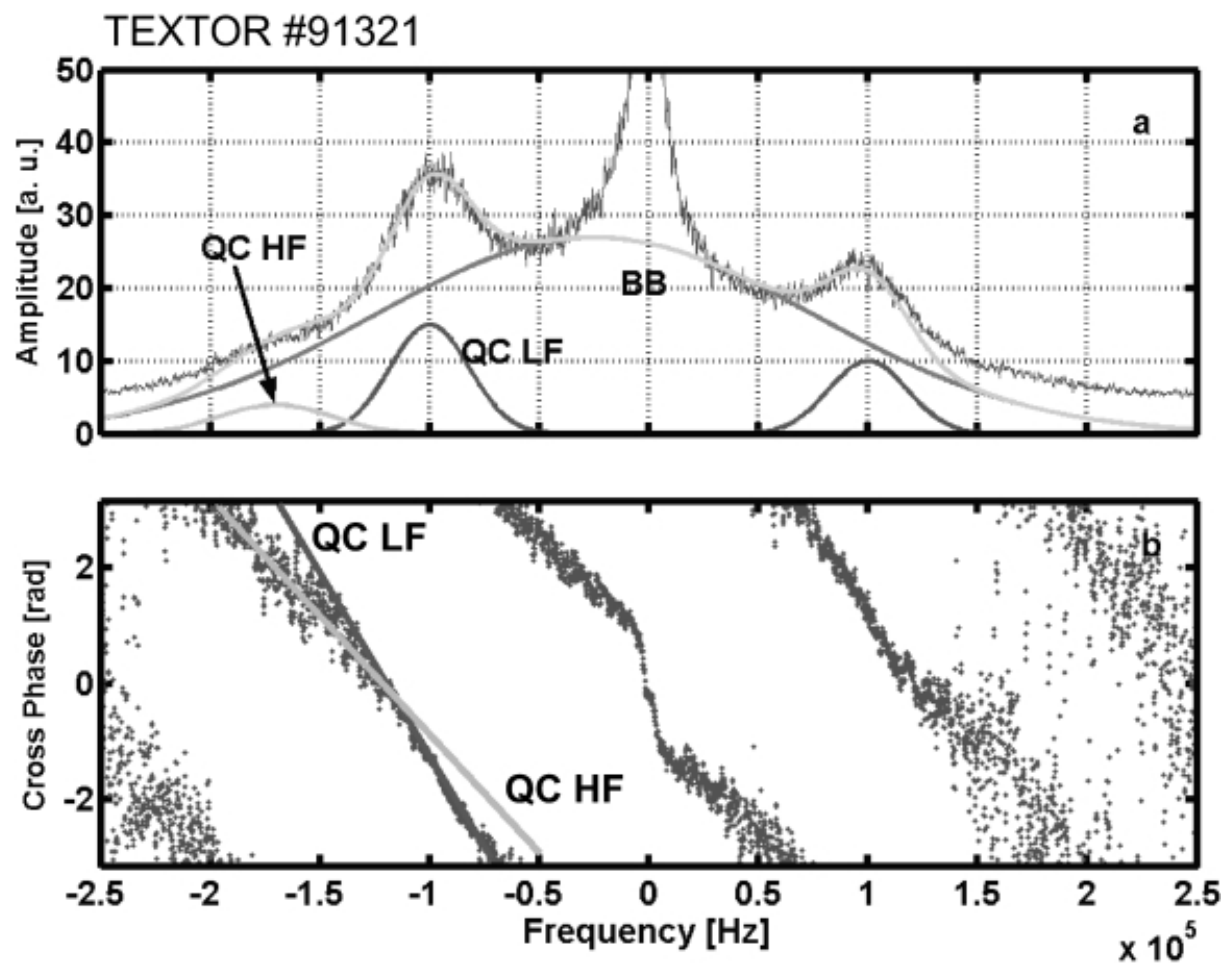

Fig. 9. Amplitude spectrum and cross phase for pulse 91321. The broadband (BB) and different quasi-coherent (QC) structures are visible in the amplitude spectrum. The QC structures show different propagation velocities as seen on the different slopes in the cross-phase spectrum (LF = low frequency).

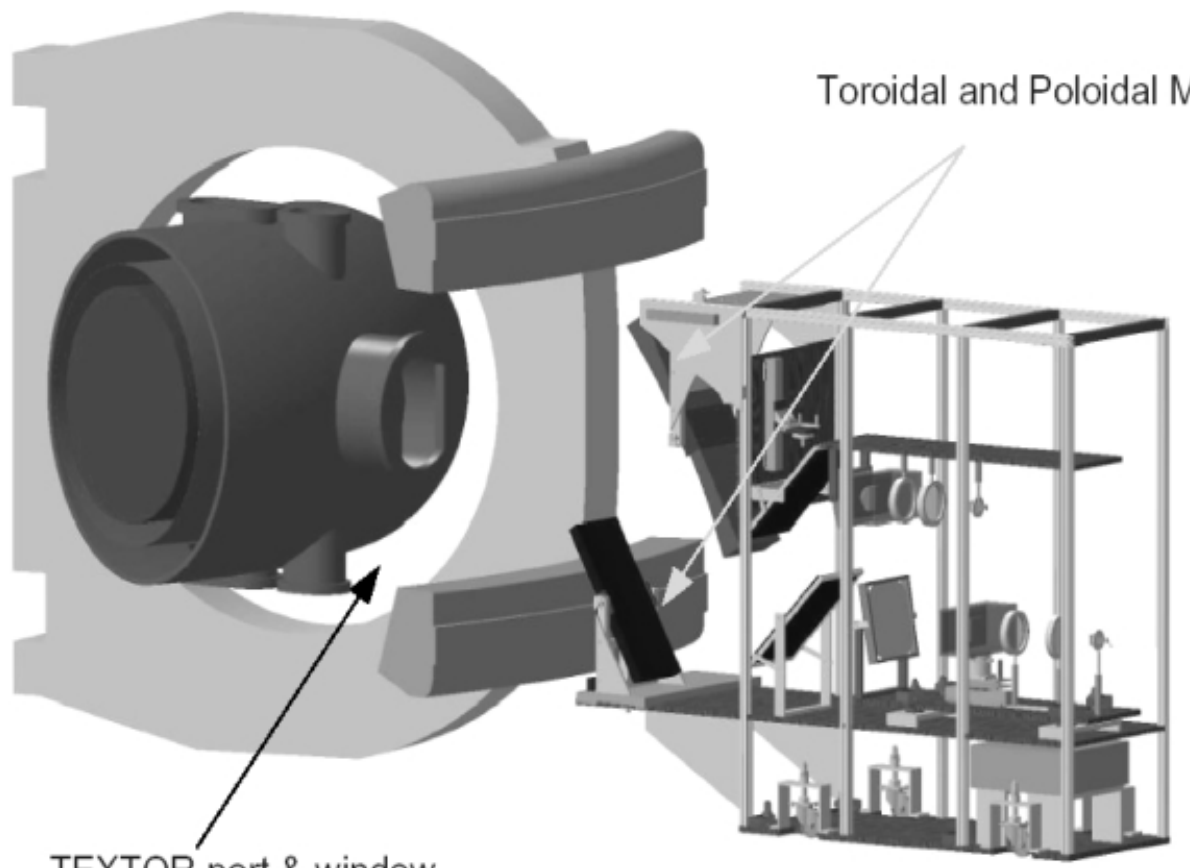

TEXTOR port \& window

Fig. 10. Computer-aided-design drawing of the combined ECEI/MIR system at TEXTOR. 
combines the advantages of wideband radiometer and "classical" ECEI systems to provide a true 2-D image [with a total of 128 channels, arranged in a matrix of 8 (horizontal) $\times 16$ (vertical) sample volumes], of $T_{e}$ fluctuations, corresponding to a total area of $8 \times 16 \mathrm{~cm}^{2}$ of a poloidal cross section. The radial position of the sample area can be shifted between plasma shots by tuning the local oscillator frequency to cover the plasma region of interest. This system was installed in December 2003 and has so far been used to make detailed 2-D studies of the temperature variations during the precursor and crash phase of sawteeth in the plasma core (see Fig. 11 on p. 227).

MIR carefully matches the probing beam of the reflectometer to the position and curvature of the reflecting surface and collects the reflected radiation in as large as possible an acceptance angle. The detailed testing of the optical system for the MIR was performed with known targets (corrugated metal surfaces) and compared with the response of a conventional 1-D system. ${ }^{23}$ It was shown that detailed structures of the reflecting surface could be recovered by imaging the reflected signal on a detector array positioned at a location relatively far away from the reflecting surface $(\sim 2 \mathrm{~m}$ in our case). This was impossible to achieve with a conventional 1-D reflectometer, where correlation between measurement and surface structure decreased rapidly over distance between reflecting surface and receiver antenna. The MIR system was installed on TEXTOR in September 2003. Evaluation of the experiments is in progress.

\section{III.D. Fast Ion CTS}

The diagnostics that have been discussed hitherto are aimed at studying the detailed evolution of the electron temperature and density profile. However, to obtain a coherent picture of the plasma confinement, it is also necessary to diagnose the parameters of the various ion populations. One particular group of ions that is very difficult to diagnose with good spatial and temporal resolution is the population of fast ions. In future burning fusion plasmas, these ions are principally the products of fusion reactions. This population will significantly impact the dynamics of the plasma, and it is essential for sustaining the burn that the fast ions be confined in the plasma while they slow down and heat the bulk plasma. For these reasons it is very important to develop adequate diagnostic tools to observe these ions on present confinement devices. In medium-sized tokamaks, like TEXTOR, the fast ions can be generated by auxiliary heating, in particular, by ion cyclotron resonance heating (ICRH).

Apart from the general interest in the development of fast ion diagnostics for the future generation of fusion devices, it is also important to have a better understanding of the fast ion dynamics, to verify or to falsify theoretical models on fast ion dynamics, to see whether fast ions are expelled from the plasma core by sawtooth instabilities, etc. Also, specific issues of ICRH physics need observations to test the existing models.

To diagnose the confined fast ions in TEXTOR, a pilot fast ion collective TS (CTS) system was installed in 2000 and was operated at TEXTOR as a collaborative effort between FOM-Institute for Plasma Physics Rijnhuizen (FOM-Institute), The Netherlands, and the Massachusetts Institute of Technology. ${ }^{24}$ The basic principle of this diagnostic is that one scatters the electrons surrounding each ion in its Debye sphere. Because of this collective effect of the electrons, it is possible to study the ion population. This system obtained many useful data on fast ion dynamics and proved for the first time the viability and strengths of this type of fast ion diagnostic. These data are currently being analyzed and prepared for publication. The CTS diagnostic probes the plasma with millimeter waves generated by a gyrotron producing $100-\mathrm{kW}$ radiofrequency power at $110 \mathrm{GHz}$ for an integrated pulse length of $0.2 \mathrm{~s}$. Scattered radiation, with information on the localized velocity distribution of the confined fast ions, is collected by a steerable antenna and spectrally and temporally resolved in a heterodyne receiver.

In 2002 and 2003 the CTS system transmission line upgrades were designed and constructed at Ris $\varnothing$ National Laboratory. ${ }^{25}$ The main improvements are a steerable ( $\pm 30 \mathrm{deg})$ quasi-optical antenna, a universal polarizer, and a quasi-optical transmission line allowing us to locate the electronics $4 \mathrm{~m}$ from the tokamak. The quasioptical transmission line consists of three in-vessel mirrors, a circular corrugated waveguide mounted with a fused silica window, four out-vessel mirrors, the two polarizer plates, and a corrugated scalar horn feeding the signal to the detector system electronics. The CTS antenna seen from inside TEXTOR is presented in Fig. 12. Two motors, one rotating the mirror fork and the other tilting the mirror, respectively, control the flat steerable antenna mirror. In conjunction with the steerable electron cyclotron resonance heating $(\mathrm{ECRH})$ launcher, it is possible to study scattering over different angles and at different locations and to observe any anisotropies in the fast ion population.

While the probe power required for a millimeterwave fast ion CTS diagnostic generally is on the order of $100 \mathrm{~kW}$ to $1 \mathrm{MW}$, the power in the scattered spectrum is on the order of $1 \mathrm{nW}$. Furthermore, the background noise is 10 to 1000 times the CTS signal. ${ }^{24}$ These circumstances set high requirements for the electronics in a CTS receiver. In order to meet these requirements better, the receiver electronics of the pilot project TEXTOR CTS system have been upgraded. A diagram of the receiver is presented in Fig. 13.

Some of the main components are the notch filters, following immediately after the horn that interfaces the quasi-optical transmission line and the receiver box. The notch filters attenuate the gyrotron stray light by damping a $250-\mathrm{MHz}$ band around $110 \mathrm{GHz}$ by $120 \mathrm{~dB}$. After the waveguide components, the signal is down-converted 


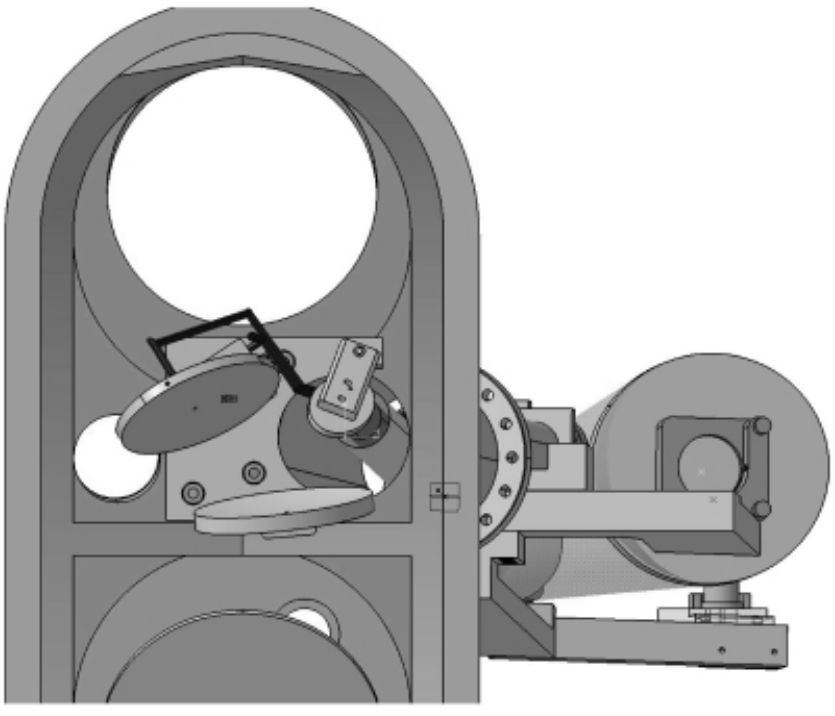

Fig. 12. The TEXTOR CTS antenna seen from inside TEXTOR. The steerable antenna mirror is to the left. by $100.5 \mathrm{GHz}$ and then split into three frequency bands by a triplexer (actually two diplexers combined). One band contains the central part of the spectrum where most of the spectral energy is, while the two other bands contain, respectively, the lower and upper spectral ranges. The splitting is done in order to avoid saturation of the amplifiers, which amplify the signal in each frequency band by 60 to $70 \mathrm{~dB}$, and to spectrally separate the sensitive wings of the CTS spectrum from the stray light from the probe. After amplification, the signals enter frequency-multiplexing filter banks. The signal strengths in the channels of the filter banks are detected by diodes, the output of which are digitized and stored by the data acquisition system. The amplifiers were carefully chosen to minimize gain saturation. Typical specifications as represented by the upper wing of the IF spectrum are frequency band 10 to $16 \mathrm{GHz}$, gain 35 to $40 \mathrm{~dB}$, output power of $+15.9 \mathrm{dBm}$ at the $1 \mathrm{~dB}$ gain compression point, and a noise figure below $1.8 \mathrm{~dB}$.

Finally, a new data acquisition system has been constructed with 40 channels sampled at 100 kilosamples

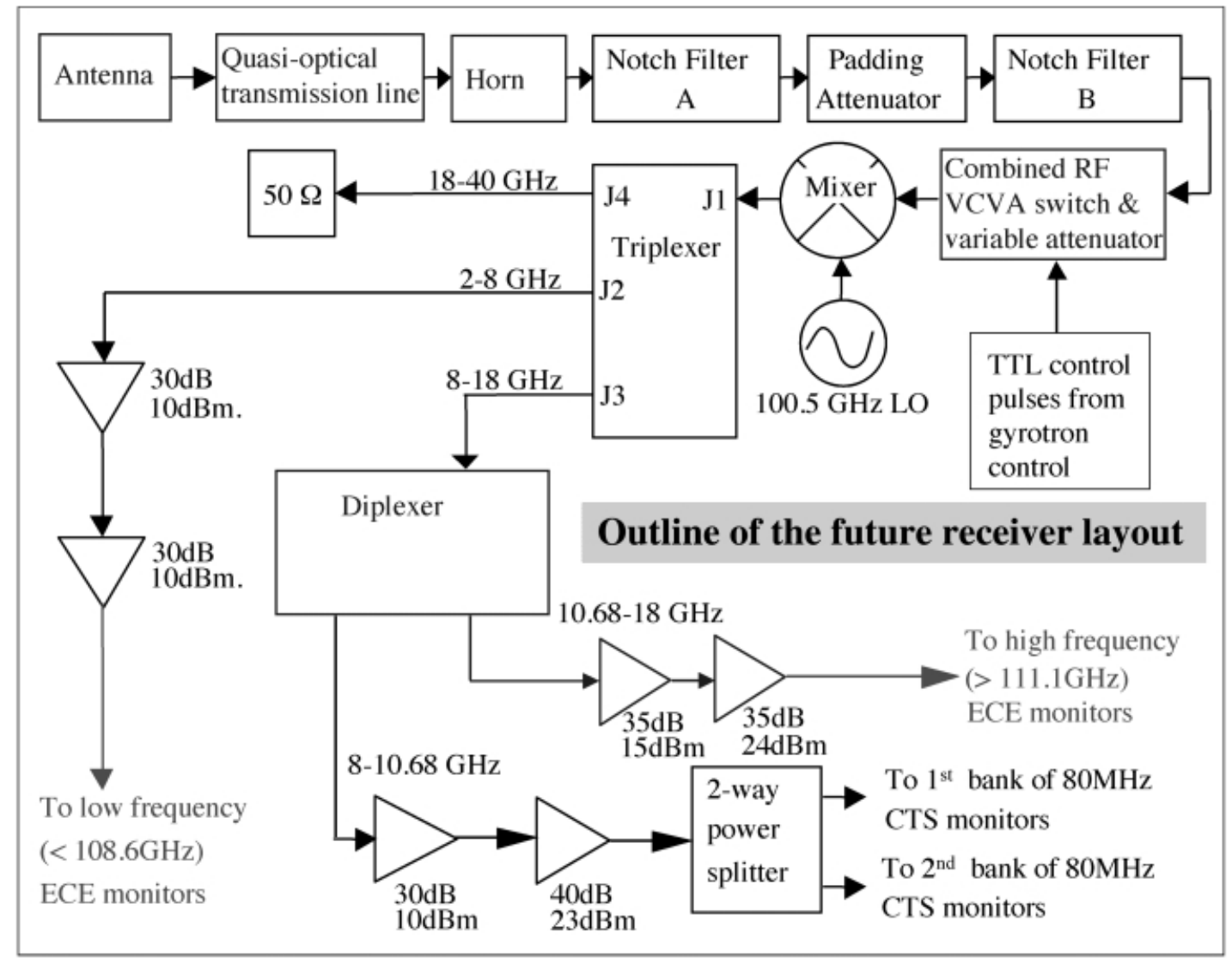

Fig. 13. Layout of the electronics in the TEXTOR CTS receiver. The first three components (top left), VCVA switch, and diplexer are new for the upgrade of the system. 
with a 24-bit resolution (effectively about 20 bits taking noise into account). The receiver and data acquisition system will allow complete coverage of the double sideband scattered spectrum for localized $(<10 \mathrm{~cm})$, timeresolved $(\sim 4 \mathrm{~ms})$ ion velocity distribution measurements, corresponding to an ion deuterium energy range of $\sim 0.5$ to $200 \mathrm{keV}$.

The diagnostic was installed in the summer of 2004. Full system tests and first operation have been started at the publication time of this paper.

\section{BEAM-AIDED SPECTROSCOPY AND NPA}

In the past several particle diagnostics have been implemented on TEXTOR. The first actual scientific collaboration between the FOM-Institute and the Institut für Plasmaphysik started in the late 1980s, almost a decade before the TEC collaboration agreement was signed. This collaboration was focused on an active beam scattering system to measure the ion temperature. ${ }^{26}$ The so-called Rutherford scattering system featured a $35-\mathrm{keV}$ neutral helium beam that was injected vertically into the plasma from the bottom. ${ }^{27}$ The forward scattering by the fuel ions over an angle of 3 to 8 deg was observed by means of an especially designed neutral particle analyzer. ${ }^{28}$ The system was able to determine the fuel ion temperature profile in TEXTOR from the Doppler broadening of the scattering distribution with a spatial resolution of $\sim 10 \mathrm{~cm}$ on a shot-to-shot basis. ${ }^{29}$ The time resolution of the system was even good enough to study sawtoothing of the ion temperature. ${ }^{30}$ By tilting the analyzer in the toroidal direction, the toroidal rotation of the plasma ions could also be measured. ${ }^{31}$ Despite the success of the Rutherford scattering system, it was decided to dismantle it in 1994: On one hand the maintenance of the system was rather labor intensive while on the other hand the ion temperature measurement could be more straightforward and with a higher spatial resolution by means of charge exchange (CX) recombination spectroscopy (CXRS).

From the beginning of TEXTOR operation, neutral particle analysis (NPA) has been used to determine the ion temperature. ${ }^{32}$ This has been the standard method to measure the ion temperatures, before $\mathrm{CX}$ spectroscopy and X-ray spectroscopy were developed. In NPA the energy spectrum of fast neutral fuel atoms is measured. These fast atoms are produced in the plasma core by CX of neutral atoms from the wall with the hydrogenic fuel ions in the plasma. Low concentrations of neutral atoms are present in the plasma, mainly by diffusion of neutrals from the plasma edge to the plasma center.

The fast neutralized fuel ions can escape from the plasma since they are not confined by the magnetic fields. In the neutral particle analyzer that has been used at TEXTOR, the escaping fast neutrals are first ionized in a gas-stripping cell. The ions are then deflected by an electrostatic field and guided to particle detectors. The thus- measured energy spectrum was corrected for reabsorption along the line of sight due to the interaction of the plasma with the escaping neutrals. The spectra at TEXTOR could be measured along different lines of sight. The temperature profile was then reconstructed by comparing the experimental spectra with EIRENE code calculations. In NPA the high-energy tail of the distribution function is measured, which is sensitive to slight deviations due to drifts in the ripple of the toroidal magnetic field, which can produce considerable deviations from a Maxwellian distribution. The NPA diagnostic was disassembled from TEXTOR when the flange was needed for a feedthrough of the new dynamic ergodic divertor (DED).

\section{IV.A. Charge Exchange Recombination Spectroscopy}

To determine profiles of various plasma parameters, CXRS has become a standard diagnostic on tokamaks equipped with a neutral beam. The principle of CXRS is that fully stripped ions in the plasma core have CX reactions with atoms from a neutral beam. The electrons are captured in excited states and cascade downward while emitting radiation. The spectrum of the emitted radiation gives information about the ion species. The Doppler broadening and Doppler shift of the spectral lines yield information on the ion temperature and plasma rotation, respectively. On TEXTOR two separate CXRS systems are operational. One uses one of the two heating beams [1.5 MW; $50 \mathrm{kV}$ of $\mathrm{H}, \mathrm{D}$, or $\mathrm{He}$; full-width at halfmaximum $(\mathrm{FWHM})=20 \mathrm{~cm}$; tangential injection]. ${ }^{33}$ The other system is based on a diagnostic neutral beam ${ }^{34}$ $(50 \mathrm{kV} ; 1$-A equivalent neutral current; FWHM $=5 \mathrm{~cm}$; radial injection), which is modulated to separate active and passive parts of the spectrum. The diagnostic beam allows us to obtain CXRS data also in non-neutral-beaminjection (NBI) heated discharges (ohmic, ECRH, and ICRH).

The optical systems viewing the heating beam are combined with the motional Stark diagnostic (see Sec. IV.B). The optics are mounted in a retractable vacuum tube, installed directly in the vessel, without any window. When the diagnostic is not in use, it is retracted behind a vacuum valve. Fiber bundles are used to transfer the light out of the tokamak toward three remotely located spectrometers. In this way, three different impurities can be measured simultaneously at 20 radial positions. The spectrometers used are of the Littrow type, with $F$-number $=4.5$ and $f=0.75 \mathrm{~m}$. Two of them are equipped with Wright CCD cameras and are able to record a full profile in $40 \mathrm{~ms}$. The third spectrometer features a Pixelvision Pluto camera, which is able to measure 20 spectra (one profile) in $<5 \mathrm{~ms}$.

In standard operation, the beam emission (Balmeralpha radiation) is always measured to make possible a more accurate determination of the impurity concentrations. These can be obtained from the ratio of the CXRS 
emission and the beam emission and the ratio of the relevant emission rate. The cumbersome absolute calibration of the spectrometers, which might change in time because of the deposition of the optical elements with hydrocarbon layers, and the calculation of the beam attenuation are then not necessary anymore.

Ion temperature measurements are routinely done using CXRS at the Carbon-VI line at $529.0 \mathrm{~nm}$. Some typical measurements with CXRS at the heating beam are shown in Fig. 14. The measurements are for different phases in a single discharge. When no neutral beam is active (Fig. 14a), the emission is from passive processes at the plasma edge: excitation by impact reactions and (passive) CX reactions with neutrals from the wall. A narrow spectrum is observed representative of the low temperature at the edge. When the neutral beam is switched on (Fig. 14b), additional emission from the (active) CX process of the fully ionized carbon ions with the neutrals from the beam is recorded. When neon is injected into the discharge (as is often done in TEXTOR to achieve the RI-mode; Fig. 14c), some neon lines complicate the carbon spectrum. Nevertheless, a reliable fit of the spectrum is possible. Figure 15 shows contour plots of the ion temperature and the toroidal rotation measured with the CXRS system on the heating neutral beam for a plasma in which the DED and ECRH are applied.
The CXRS observation of the diagnostic hydrogen beam consists of two systems for the plasma center and edge (Table I). The optics are installed in movable tubes inserted into the TEXTOR vacuum chamber to increase the observation regions. The detected light is transferred via glass fibers to the remote spectrometers. Both systems contain about 20 radial channels and have a time resolution depending on the CX line intensity (typically $100 \mathrm{~ms}$ ). The edge observation has recently been equipped with a high-resolution spectrometer for precise measurements of the poloidal plasma rotation by the Doppler shift. Additional lines of sight, which look from the opposite side, provide an in situ determination of the unshifted wavelength.

\section{IV.B. Motional Stark Effect}

The current density (or safety factor) profile at TEXTOR is measured with a newly installed motional Stark effect (MSE) diagnostic. This measurement relies on the spectral shape of the Balmer-alpha emission of the hydrogen or deuterium neutral beam. Injected fast neutral atoms with velocity $v_{b}$ moving in a magnetic field $B$ experience a Lorentz electric field $E_{l}=v \times B$, which causes a Stark splitting of the emitted Balmer-alpha line. This light is either polarized parallel to the electric field (pi polarized component, for the $\Delta m=0$ transition,

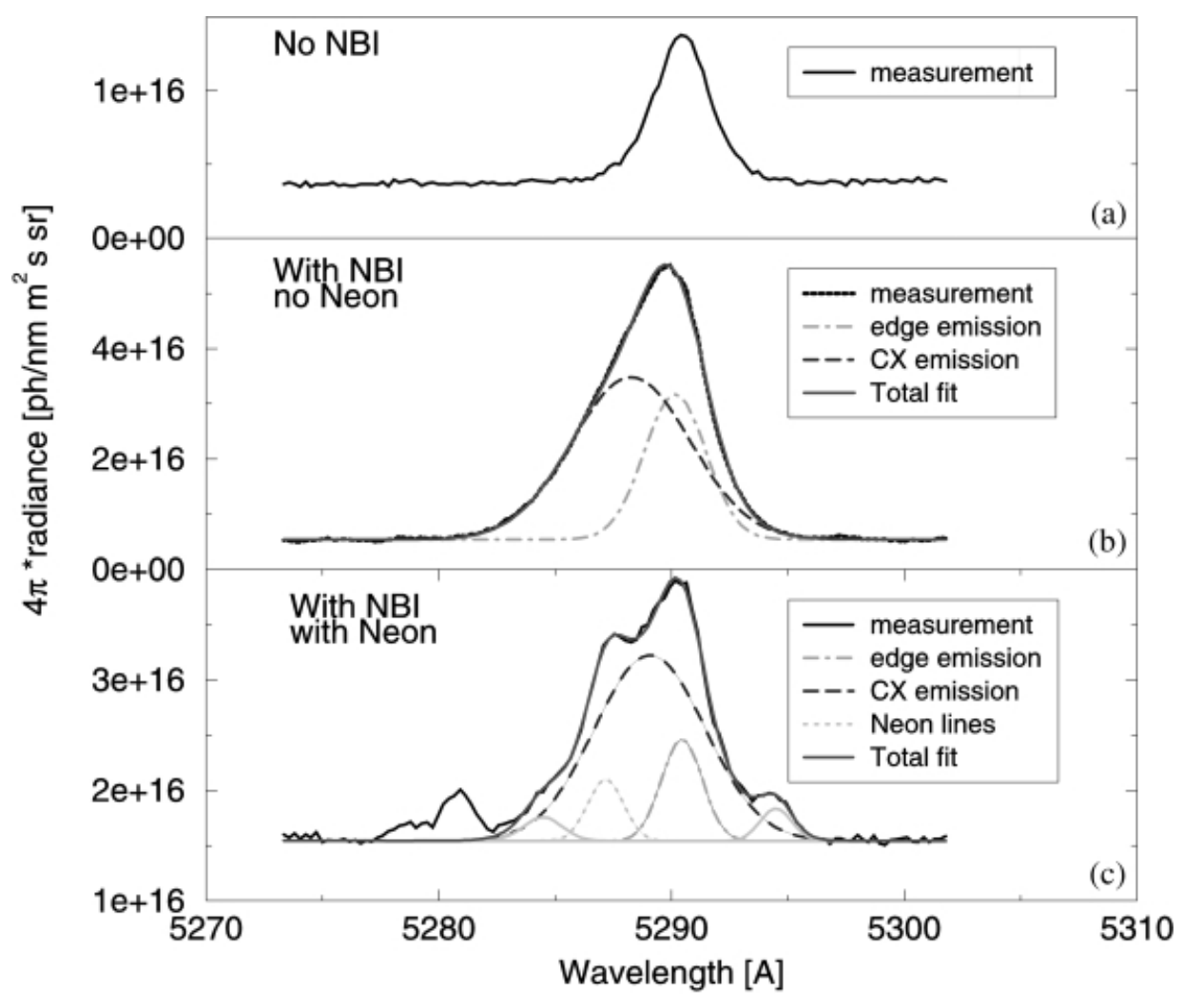

Fig. 14. Sample spectrum of Carbon-VI $(n=8 \rightarrow n=7$ at $529.0 \mathrm{~nm})$. 

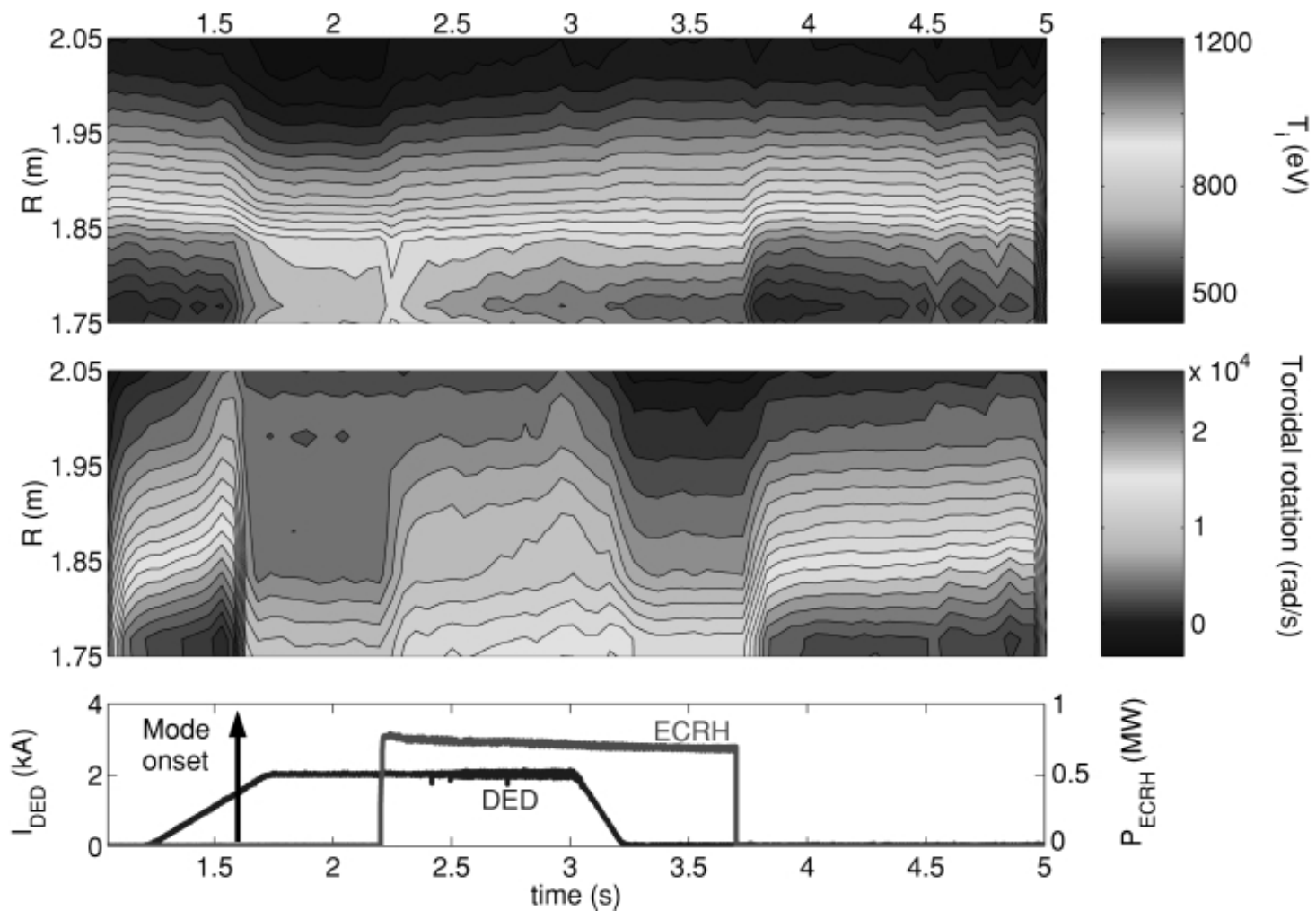

Fig. 15. Contour plots of the ion temperature (top) and toroidal plasma rotation (middle) for a plasma in which the DED and ECRH are applied (bottom). An $m=2$ mode sets in at $\sim 1.6 \mathrm{~s}$.

$m$ being the projection of the total orbital momentum on the electric field direction) or polarized perpendicular to the electric field (sigma polarized component, for $\Delta m=$ \pm 1 ). The geometry of the measurement system secures clear separation of the plasma emission from beam emission by the Doppler shift. (The three beam components are separated from each other as well.) Now, by determining either the polarization direction of the pi or sigma components, or the ratio between the pi and sigma intensities, the direction of the magnetic field and hence the pitch of the field lines can be determined. If in addition the radial position of the measurement is included in the analysis, the $q$ profile or the current density can be determined.

At most existing tokamaks, narrow band interference filters are used in the MSE systems to detect one of the lines and measure its polarization direction by photoelastic modulators. In contrast, in the MSE system operational at TEXTOR, the full polarization spectrum of the emitted light is measured. ${ }^{35,36}$ The advantages of exploiting the full spectral information are obvious:

1. The system is insensitive to variations in beam velocity or magnetic field.

TABLE I

Properties of the Observation System of the Diagnostic Beam

\begin{tabular}{|l|c|c|}
\hline & Center & Edge \\
\hline Covered radial range $R-R_{0}(\mathrm{~cm})$ & -12 to 27 & 20 to 49 \\
1 to 2 \\
Radial resolution $(\mathrm{cm})$ & 2 to 8 & $0.07 / 1 \mathrm{~m}$ \\
Numerical aperture of spectrometer & $0.07 / 0.75 \mathrm{~m}$ & 0.7 \\
Spectral resolution $(\AA / \mathrm{mm})$ & 4.4 & Dalsa $1024 \times 1024$ \\
CCD detector & Dalsa $256 \times 256$ & $800 / 120$ \\
Fiber diameter/slit width $(\mu \mathrm{m})$ & $800 / 100$ & \\
\hline
\end{tabular}


2. The Doppler shift is measured as well, allowing one to determine the observation volume directly from the spectrum.

3. The polarization is measured at several lines simultaneously, increasing the accuracy of the deduced direction of the magnetic field

4. The Stark splitting is obtained allowing one to compute the magnitude of the magnetic field.

5. If in addition to the motional Stark electric field a radial electric field is present in the plasma, this can, albeit with limited accuracy, be determined as well by comparing the polarization direction of two different energy components of the neutral beam.

A trade-off of spectra polarimetry compared to the traditional approach is that the optical throughput is reduced. However, in the present case this does not limit the time resolution since the signal level is high enough. A second trade-off is that the data analysis of full spectra polarimetry is more complex.

The collection optics for the MSE system has already been described in Sec. IV.A. Important features of the MSE system are that the light impinges directly on the SFL-6 prism surface with the Verdet constant equal to zero (to avoid polarization changes in the optical path due to the high magnetic field). Then, it passes through a polarizer with the polarization angle set to $45 \mathrm{deg}$, such that the pi and sigma components have a comparable intensity, which leads to the smallest error bar for the measured intensity ratio and the highest sensitivity to changes in the magnetic pitch angle.

The lines of sight are tangential to the magnetic flux surfaces for the best radial resolution. The light is collected by three rows of 20 channels (the middle one crosses the equatorial plane) covering $0.45 \mathrm{~m}$ of the low-field side of the tokamak major radius $\left(R_{0}=1.75 \mathrm{~m} ; a=\right.$ $0.46 \mathrm{~m})$ and crosses the magnetic axis. The spatial resolution of the measurement is $\sim 3 \mathrm{~cm}$ with a channel-tochannel separation of order of 1.5 to $2 \mathrm{~cm}$.

An example of a measured MSE spectrum is shown in Fig. 16 along with the fitted theoretical curve. Two $q$ profiles measured in a single TEXTOR discharge, but at different values of the plasma current, are depicted in Fig. 17. The $q$ values in Fig. 17 have been derived from fits to the spectra. The curves in Fig. 17 are quadratic $q$ profiles with the position of the $q=1$ radius and the value of $q$ at the edge as constraint.

\section{PASSIVE SPECTROSCOPIC DIAGNOSTICS}

The performance of magnetically confined fusion plasmas is strongly related to the content and transport of plasma impurities, which include all elements in the plasma apart from the hydrogen fuel. Impurities are released to the plasma via plasma-wall contact, produced

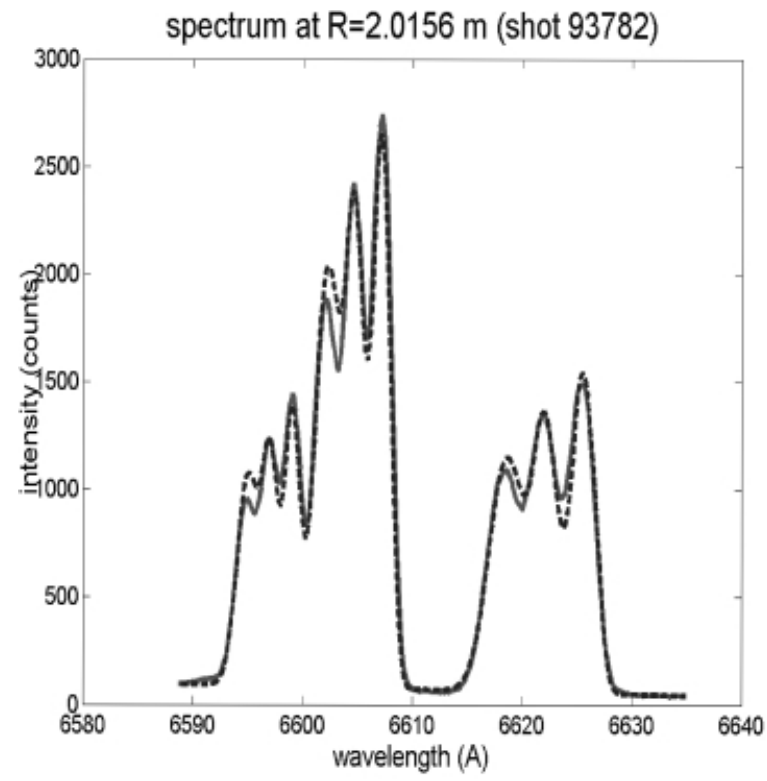

(a)

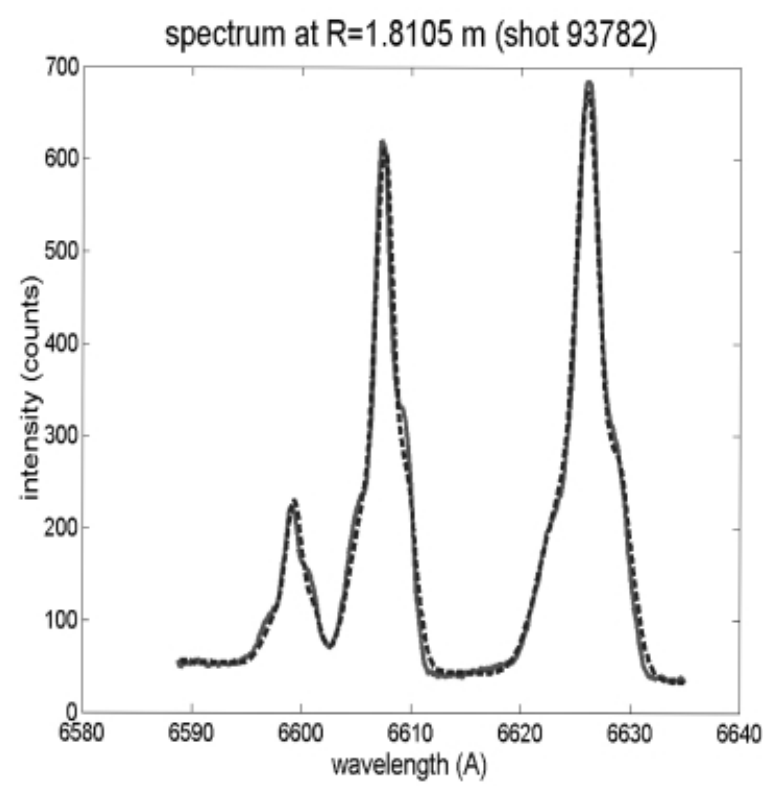

(b)

Fig. 16. Typical spectra in (a) the plasma core and (b) edge as recorded by the MSE diagnostic (full line) and the theoretical description of the spectrum (fit) by the dashed line. The three groups of emission correspond to the full, half, and third energy components of the neutral beam. Within each group the ratio of sigma (center line) to pi (left and right lines) intensities provides a measure for the magnetic pitch angle. 


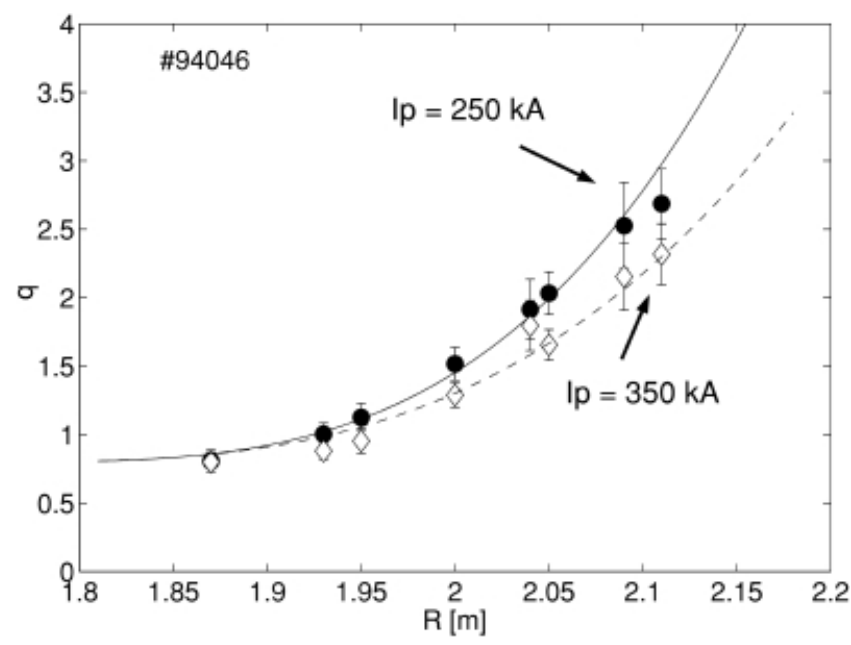

Fig. 17. Two $q$ profiles measured in the same plasma discharge but at different values of the plasma current of 250 and $350 \mathrm{kA}$, respectively.

by fusion reactions (helium) or added artificially to modify the plasma radiation properties. The most fundamental experimental access to identify impurity species is the spectroscopic measurement of their characteristic line radiation. Spectroscopy in the vacuum ultraviolet (VUV) wavelength range (10 to $200 \mathrm{~nm}$ ) with moderate spectral resolution mainly serves to characterize the relative abundances as well as the transport properties of impurities based on the measurement of their most intense spectral lines. Tomographic measurements in the X-ray wavelength range $(0.3$ to $10 \mathrm{~nm})$ enable the reconstruction of radial emissivity and density profiles of impurities. Highresolution X-ray spectroscopy can be used to determine plasma properties such as the ion and electron temperature as well as the ion drift velocity based on a sophisticated analysis of the fine structure of the measured spectra.

\section{V.A. VUV Spectroscopy}

Three VUV overview spectrometers have been implemented at TEXTOR to study the behavior of impurities in the plasma. The two Survey Poor Resolution Extended Domain ${ }^{37}$ (SPRED) channels cover the wavelength ranges of 10 to $33 \mathrm{~nm}$ and 15 to $115 \mathrm{~nm}$, respectively, while a near-normal incidence spectrometer is used in the wavelength range from 58 to $125 \mathrm{~nm}$. Various elements from hydrogen $(Z=1)$ up to zirconium $(Z=40)$ and their ions have been clearly identified based on the measurement of their most prominent spectral lines. The high time resolution of the instruments of $1 \mathrm{~ms}$ (Ref. 38) allows one to perform transient impurity injection experiments and to derive the radial transport properties of the injected impurity based on an analysis of the time evolution of spectral lines from different ionization stages. For more details we refer to Ref. 39.

\section{V.B. X-Ray Diagnostics}

\section{V.B.1. Ultra-SXR Tomography}

The aim of the ultra-soft-X-ray (SXR) tomography system on TEXTOR is to measure 2-D profiles of the local emission coefficients of line radiation emitted by impurity ions..$^{40,41}$ It uses a tomographic algorithm to reconstruct the local emission from the line-integrated brightness measured by five pinhole cameras. The cameras are placed symmetrically around a poloidal cross section of the torus in order to obtain the best possible tomographic reconstruction. To have the widest possible viewing fan, the cameras are mounted on pneumatic drivers such that during their operation the camera heads are positioned just inside the vacuum vessel. When not in operation, the cameras are retracted and positioned behind a closed vacuum valve (see Fig. 18). Multilayer mirrors are used to select one or more specific emission

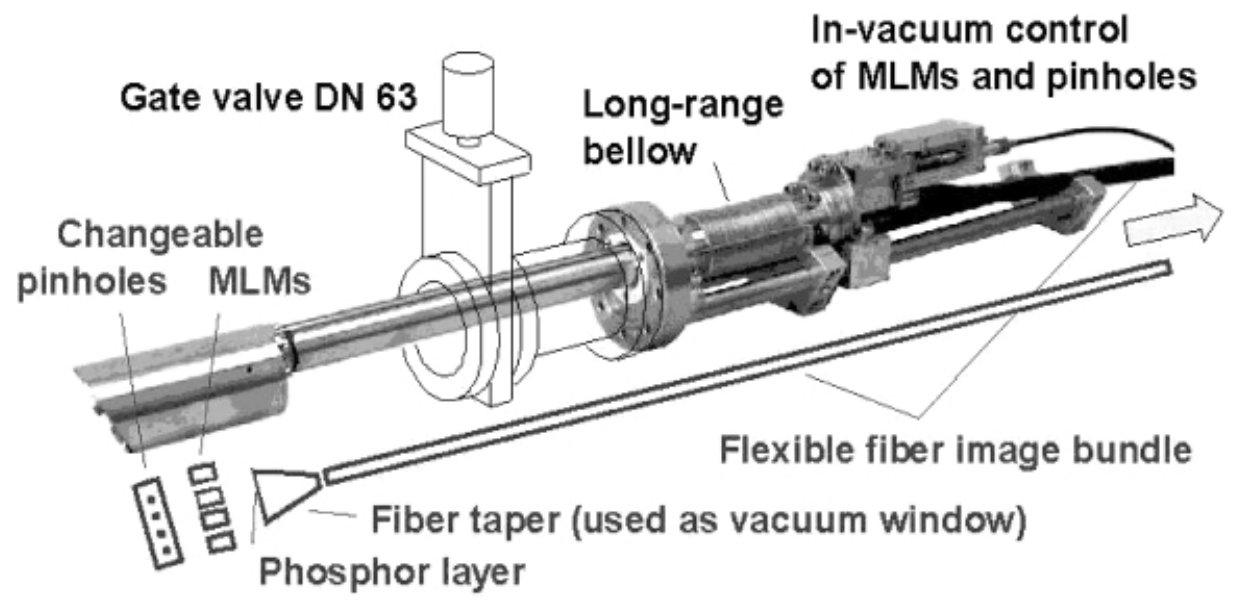

Fig. 18. Photograph of one of the retractable MLM cameras of the ultra-SXR tomography system. 
lines. Several ionization states of the elements $\mathrm{Ar}, \mathrm{Ne}, \mathrm{B}$, $\mathrm{C}$, and $\mathrm{O}$ can be selected (wavelength range: 0.3 to $12.5 \mathrm{~nm}$ ). Each camera features in total up to five different multilayer mirrors and five different pinhole apertures. Both can be selected remotely. The time resolution of the tomography system is $20 \mathrm{~ms}$. From the 2-D local emission profiles, the impurity density profiles can be derived using some additional data (electron temperature, electron density, and atomic data) from other diagnostics. Table II lists the parameters of the multilayer mirrors for a number of specific impurity lines to which the system can be tuned.

\section{V.B.2. X-Ray Spectroscopy}

$\mathrm{X}$-ray spectroscopy is one of the basic diagnostic techniques to obtain information on the high-temperature plasma. It has been used on all major tokamaks. On TEXTOR, a combined X-ray spectrometer/polarimeter for He-like argon was installed in 1997 in order to determine the central plasma parameters and to check the concepts of the theoretical description of highly ionized He-like ions. It is described in more detail in Ref. 42.

\section{MISCELLANEOUS}

\section{VI.A. Bolometry}

The radiated power of a plasma is an important figure of merit. In a clean plasma, the radiated power is dominated by radiation due to scattering of the electrons by the hydrogen ions, the so-called "bremsstrahlung." In practice, a plasma contains a certain amount of impurities, which are either released from the plasma-facing components by impinging energetic plasma particles or added artificially. Ions, which are not fully ionized, can be excited by the electrons and radiate at characteristic lines. The bremsstrahlung increases with the square of the nuclear charge of the ion; the characteristic radiation rises even with exponent 4 . Therefore, the concentration of impurities in the hot plasma core must be as low as possible to ensure that the heating rate in the center exceeds the radiation losses.

However, at the edge it is favorable if the plasma energy is converted into radiation in order to reduce the electron temperature and to keep the load on the plasmafacing components at an acceptable level. Investigation of the interplay between the properties of the plasma core and the edge and the plasma-facing components is one of the major missions of TEXTOR. Adding medium-Z impurities, which are fully ionized in the plasma core but radiate strongly at the plasma edge, can minimize the thermal load to the limiters. This radiative mantle concept has been investigated on TEXTOR in detail. ${ }^{43}$

The radiated power and the spatial distribution of the radiation are measured by bolometry. ${ }^{44}$ On TEXTOR, a simple but accurate approach is used. A thin kapton foil covered by a gold absorber is exposed to the radiation. The temperature rise of the foil is measured by the change of the resistance of a thin film resistor on the rear side of the foil. In order to increase the sensitivity, the resistor is part of a Wheatstone bridge, and the compensation resistors are of the same design but shielded against the radiation from the plasma. The bridge is operated in the alternating-current mode with synchronous demodulation to reduce noise and pickup. This type of resistive bolometer has the advantage that it can be absolutely calibrated in situ by observing the temperature rise due to a known current in the resistors.

Four bolometer cameras with $3 \times 8$ and $1 \times 12$ lines of sight, respectively, are used to measure the radiation along different rays and under different directions (Fig. 19). Tomographic techniques are applied to derive local values for the radiated power from the line-integrated

TABLE II

Parameters of Multilayer Mirrors Optimized for Specific Impurity Lines

\begin{tabular}{|c|c|c|c|c|c|c|}
\hline Ion & $\begin{array}{c}\lambda \\
(\mathrm{nm})\end{array}$ & $\begin{array}{l}\text { Multilayer } \\
\text { Structure }\end{array}$ & $\begin{array}{c}\text { Period } \\
(\mathrm{nm})\end{array}$ & $\begin{array}{c}\text { Grazing Angle } \\
\text { (deg) }\end{array}$ & $\begin{array}{l}\text { Reflectivity } \\
(\%)\end{array}$ & $\begin{array}{l}\text { Resolution } \\
\quad(\lambda / \Delta \lambda)\end{array}$ \\
\hline Ar XVIII & 0.373 & $\mathrm{Cr} / \mathrm{Sc}$ & 2.0 & 5.4 & 30 & 150 \\
\hline Ar XVII & 0.395 & $\mathrm{Cr} / \mathrm{Sc}$ & 2.0 & 5.7 & 30 & 150 \\
\hline $\mathrm{Ne} X$ & 1.213 & $\mathrm{~W} / \mathrm{Si}$ & 2.1 & 17 & 16 & 96 \\
\hline $\mathrm{Ne} I X$ & 1.345 & $\mathrm{~W} / \mathrm{Si}$ & 2.1 & 19 & 13 & 90 \\
\hline O VIII & 1.90 & $\mathrm{~W} / \mathrm{Si}$ & 2.1 & 27 & 6 & 80 \\
\hline O VII & 2.16 & $\mathrm{Cr} / \mathrm{Sc}$ & 2.1 & 31 & 7 & 150 \\
\hline Ar XIV & 2.74 & $\mathrm{Fe} / \mathrm{Ti}$ & 2.5 & 33.6 & 13 & 140 \\
\hline C VI & 3.37 & $\mathrm{Cr} / \mathrm{Sc}$ & 2.5 & 41 & 7 & 140 \\
\hline $\mathrm{C} \mathrm{V}$ & 4.03 & $\mathrm{Cr} / \mathrm{Sc}$ & 3.1 & 40 & 9 & 125 \\
\hline B V & 4.86 & $\mathrm{Fe} / \mathrm{C}$ & 3.8 & 40 & 12 & 80 \\
\hline B IV & 6.03 & $\mathrm{Cr} / \mathrm{C}$ & 4.7 & 40 & 10 & 75 \\
\hline
\end{tabular}




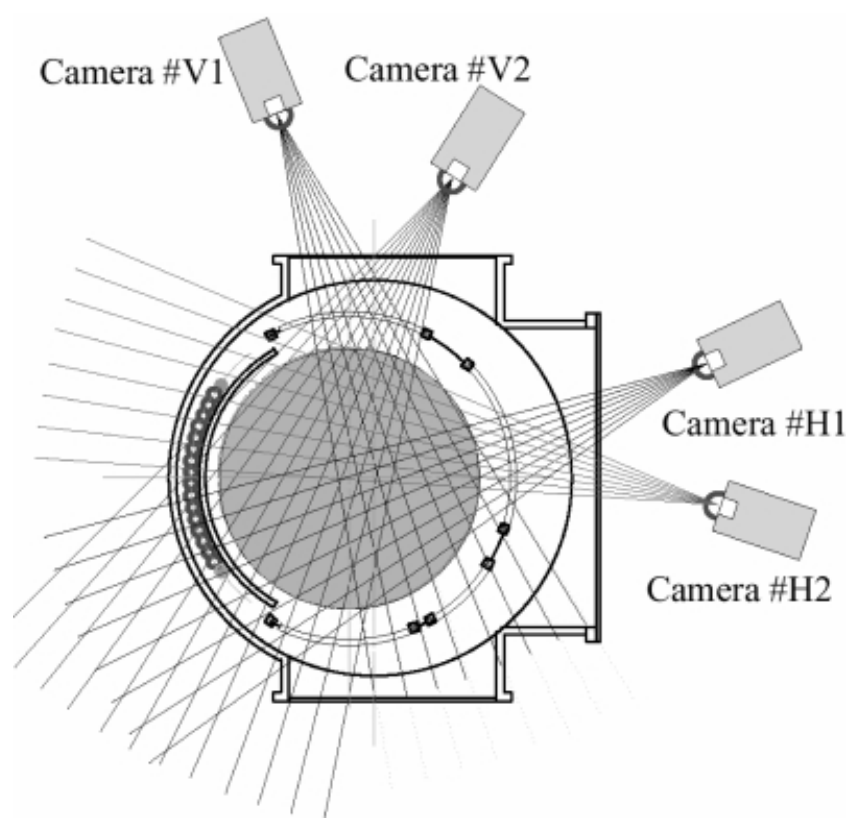

Fig. 19. Geometry of the bolometry system. measurements. Constraints are used to optimize the tomographic reconstruction; e.g., it is assumed that gradients are much stronger in the radial than in the poloidal direction. Examples of profile reconstructions are shown in Fig. 20. On TEXTOR, stable discharges with peaked emission profiles, where most of the radiation is emitted from the plasma center, as well as with a highly radiating belt at the plasma edge have been observed (Figs. 20a and 20b). At higher plasma densities, especially during DED operation, localized highly radiating regions build up at the high-field side (Fig. 20c).

In addition to the local emission, the total radiated power is deduced from the bolometer arrays, which is important for the characterization and the operation of TEXTOR at high radiation levels. In these discharges, the total radiated power approaches the power that is applied to the plasma. By adding small amounts of neon to the plasma and feedback control of both the neon flow and the heating power, stable discharges with 90 to $95 \%$ of radiation compared to the heating power have been obtained. The absolute calibration of the bolometers can be checked for plasmas with a radiation collapse, when

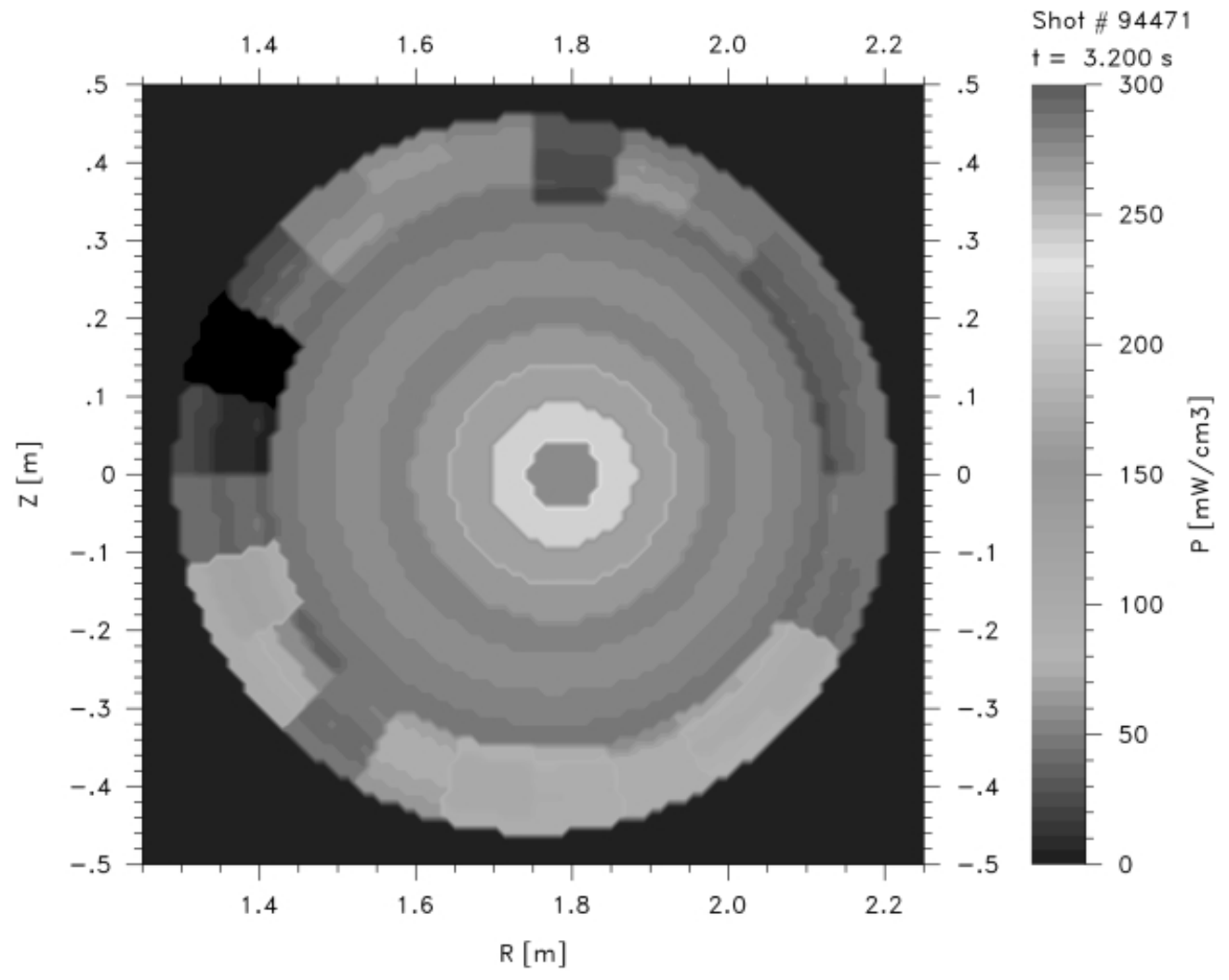

(a)

Fig. 20. (Figure continues on next page.) 


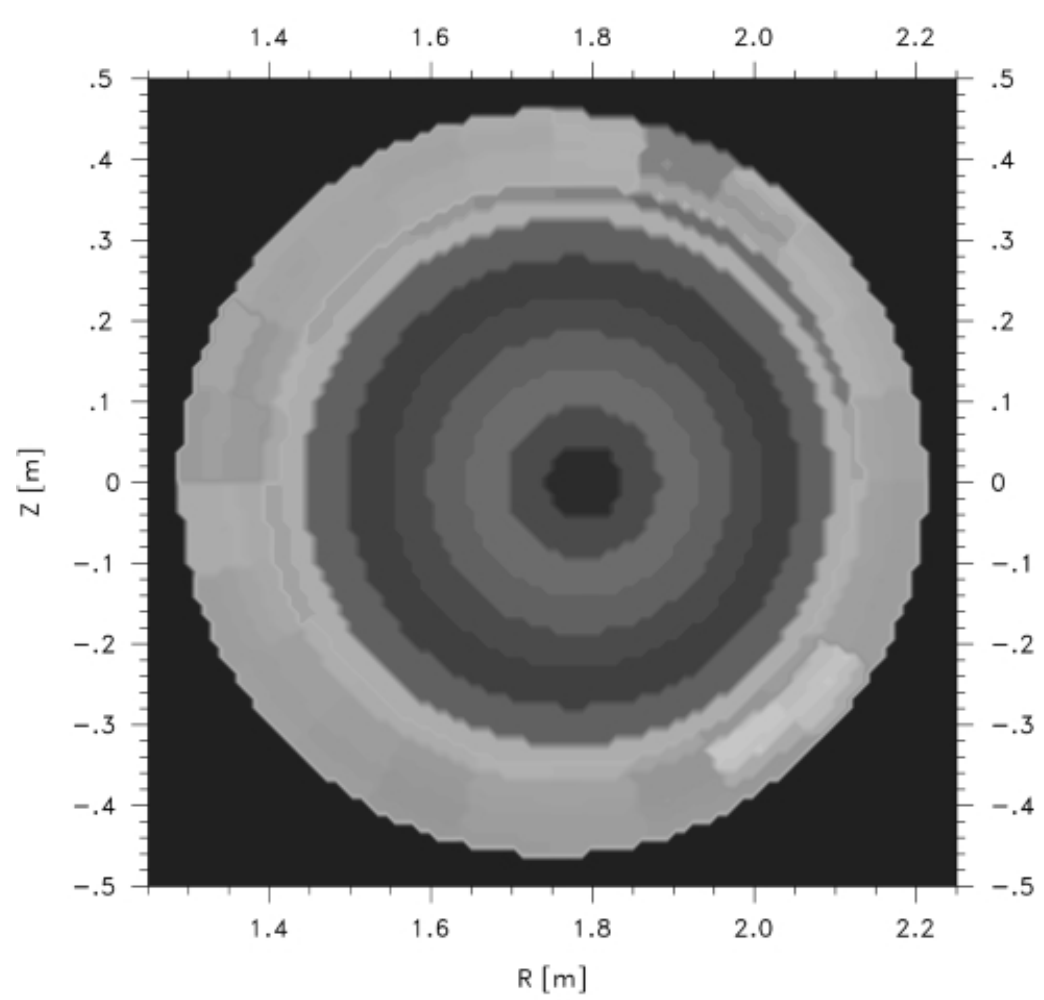

Shot \# 94091

$t=2.700 \mathrm{~s}$

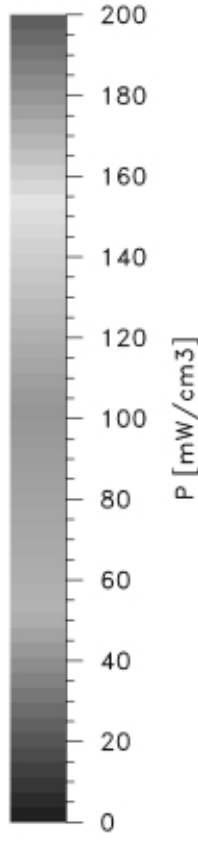

(b)

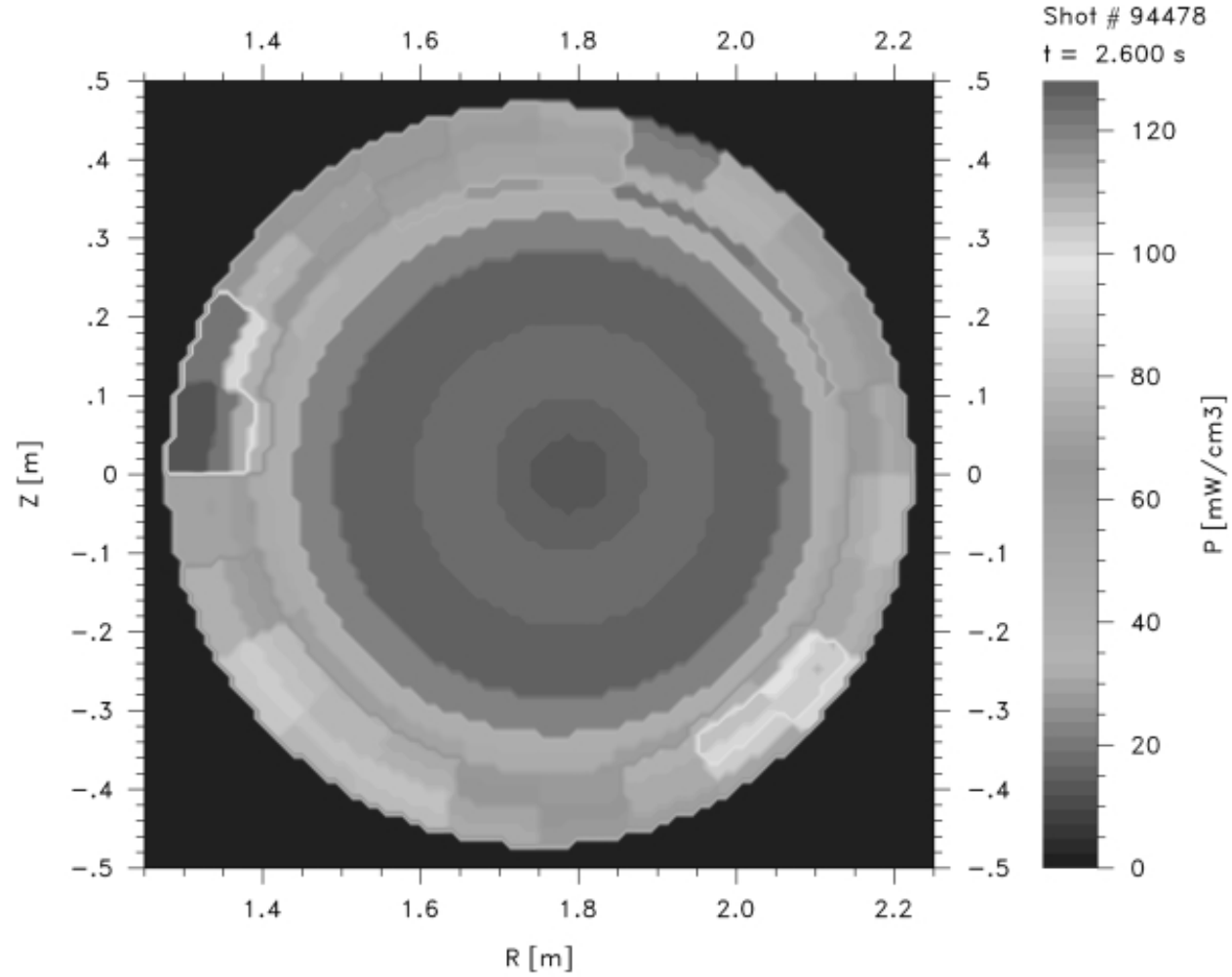

(c)

Fig. 20. Radiation profile where (a) most of the radiation is emitted from the center and (b) a symmetric radiating belt occurs at the edge. In discharges of this type, the edge plasma is cooled by radiation, while the temperature in the core stays still high. (c) A highly radiating region localized in front of the DED divertor builds up. 
the power radiated by the plasma is equal to the applied power, under the assumption that the radiation profile is toroidally symmetric.

\section{VI.B. SXR Pin-Diode Cameras}

Measurement of the SXR emission profile is a powerful technique to deduce the shapes of the internal flux surfaces; this is under the assumption that the X-ray emissivity varies only smoothly along a magnetic flux surface. In conventional radial SXR measurements, PIN diode arrays have been widely used to reconstruct plasma images by means of tomographic techniques.

On TEXTOR, four sets of SXR pin diode cameras viewing the plasma perpendicular to the torus from vertical $(2 \times 20$ channels $)$ and horizontal $(2 \times 16$ channels $)$ directions, respectively, are used to study MHD phenomena and impurity transport. Each detector is made of passivated ion-implanted silicon with an active area of $3 \times 6 \mathrm{~mm}^{2}$ and has a response time of $0.5 \mu \mathrm{s}$. The spatial resolution of the SXR camera is $\sim 2 \mathrm{~cm}$. The bandwidth of the preamplifier is $50 \mathrm{kHz}$. The data acquisition system based on PXI modules can provide sampling rates up to $200 \mathrm{kHz}$ and has a memory size of 2 megabytes/ channel. Each camera has five sets of interchangeable slits with different widths of $5,10,15,20$, and $25 \mathrm{~mm}$, respectively. Between the slit and the detector, a movable filter stick is arranged with five Be filters of different thicknesses of $0.01,0.02,0.04,0.08$, and $0.5 \mathrm{~mm}$. Therefore, the flux of SXRs can be adjusted to the dynamic range of the detectors and the amplifiers by choosing appropriate combinations of the slits and the filters.

Figure 21 shows the temporal evolution of radial line-integrated X-ray-emission profiles measured by a vertical SXR camera for a plasma heated by NBI. In this experiment, the DED is operated in the direct-current $m / n=3 / 1$ mode, where $m$ and $n$ are the poloidal and toroidal mode numbers, respectively. A strong $m / n=2 / 1$ sideband is created. When the DED coil current is increased above $0.75 \mathrm{kA}$, the rotation of the $m / n=2 / 1$ mode stops, and a nonrotating island is observed. The width of the $m / n=2 / 1$ mode derived from the flattening of the X-ray emission is $\sim 6 \mathrm{~cm}$, which is $13 \%$ of the minor radius of the plasma. When the error field induces an $m / n=2 / 1$ island in the plasma, the sawtooth instabilities are observed to disappear.

\section{VI.C. Tangential X-Ray Camera}

A tangentially viewing SXR camera system ${ }^{45}$ has been developed in a collaboration of the Princeton Plasma Physics Laboratory, the TEXTOR group, and the National Institute for Fusion Science in Japan. The aim of the tangential X-ray camera is to extend the range of the traditional radial $\mathrm{X}$-ray diode arrays.

In both diagnostics, the X-ray intensity is integrated over the lines of sight and collected by appropriate X-ray detectors. The local X-ray emission is calculated via inversion techniques. To observe small structures in the plasma by means of traditional SXR tomography, many lines of sight from different viewing directions are required. Often, assumptions need to be made about the smoothness of the structures along and perpendicular to the flux surfaces to obtain reliable reconstructions of the $\mathrm{X}$-ray emissivity. Another drawback is that the measurements are line integrated such that the contribution of small-scale structures to the signal has a large background from the rest of the plasma. A possible solution is to adopt a geometry in order to increase the integration length for the structures that are to be investigated, as is done in the tangentially viewing X-ray camera. This system is designed to increase the integration length for structures on the magnetic surfaces by observing parallel or under small angles to the local magnetic fields.

The system is based on a tangentially viewing pinhole (2-mm-diam) camera. The pinhole is covered by a $14-\mu \mathrm{m}$-thick beryllium foil. The SXR image of the plasma is converted to a visible one using a P47 scintillator (100-mm diameter). The image is transferred by a plastic fiber-optics bundle (Toray PGR-FB750, $100 \times 100$ elements) and is intensified by an electron beam-type image intensifier (Hamamatsu Photonics, V4440U-mod). The size of the image is thereby reduced by a factor of 4 . The image is further intensified by a second image intensifier (Hamamatsu Photonics, C7244) together with an image booster (Hamamatsu Photonics, C4412). It is then recorded by a fast-framing video camera (Kodak 4540MX), which determines the speed of the system. The framing rate is either $4.5 \mathrm{kHz}$ (full-frame mode) or $13.5 \mathrm{kHz}$ (half-frame mode). This makes it possible to study slow fluctuations, e.g., MHD instabilities, with high $(100 \times$ 100) spatial resolution. An example of measurements during a sawtooth event is shown in Fig. 22. From the composite pictures, several independent components are separated by a singular value decomposition method. ${ }^{46}$ The time-averaged profile (A), the components related to the sawtooth crash (B), and two $m=1$ precursor components $(\mathrm{C}, \mathrm{D})$ can be recognized. Since the $m=1$ structure is rotating, two $m=1$ components with different phases appear.

\section{VI.D. $Z_{\text {eff }}$ Measurement}

The $Z_{\text {eff }}$ profile in TEXTOR is determined from the bremsstrahlung in the wavelength range $523 \pm 1 \mathrm{~nm}$. From several diagnostics operated over a long time period, it has been found that this wavelength region is free of any emission lines. Reflections are minimized as much as possible by using carbon viewing dumps or carbon coating of parts of the vessel where carbon tiles cannot be mounted. The detection system features a CCD camera with 21 viewing chords. This makes it possible to determine $Z_{\text {eff }}$ also during transient phases. A few additional channels are equipped with photomultipliers to 
allow acquisition of the signals with a higher time resolution than that possible with the camera. From the lineintegrated signal of the central chord, the line-averaged $Z_{\text {eff }}$ value is routinely computed every $100 \mathrm{~ms}$ using information on the density and temperature profiles from the HCN interferometer and ECE diagnostics, respectively. The line-averaged $Z_{\text {eff }}$ value is available online.

\section{VI.E. Synchrotron Observation}

Runaway electrons can be confined long enough in present-day tokamak discharges to gain energies of the order of several tens of mega-electron-volts. At these energies they emit synchrotron radiation in the (near-) infrared wavelength range, which can easily be detected by thermographic cameras. On TEXTOR a unique diagnostic exploiting this synchrotron radiation has been developed. ${ }^{47,48}$ It consists of an infrared camera that views the plasma tangentially toward the direction of electron approach. This camera, an Inframetrics 760 broadband (upgraded to 12 bits), includes a liquid nitrogen-cooled $\mathrm{HgCdTe}$ detector; focusing lens; and two scanning mirrors, one horizontal and one vertical. With this camera a 2-D TV picture is generated according to the National Television System Committee (NTSC) standard. One line is scanned in $65 \mu \mathrm{s}$; a complete picture consists of 256 lines and is scanned in $16.7 \mathrm{~ms}$. One complete frame contains therefore information on space and time simultaneously. The detector is sensitive in the wavelength range of 3 to $12 \mu \mathrm{m}$, but since $\mathrm{CaF}_{2}$ optics is used, this is effectively from 3 to $8 \mu \mathrm{m}$.

The diagnostic provides a direct image of the runaway beam inside the plasma. From the spectral features the runaway energy can be obtained, the intensity of the radiation is a measure of the number of runaway electrons, and the synchrotron spot carries information on their perpendicular momentum and spatial distribution. Figure 23 gives an example of such synchrotron measurement at TEXTOR in the wavelength range of 3 to $8 \mu \mathrm{m}$ where the emission reaches its maximum for 20 - to $30-\mathrm{MeV}$ electrons.

With this diagnostic several interesting results have been reported:

1. In steady-state discharge conditions, an exponential increase in the synchrotron radiation signal is observed. This was the first evidence of the so-called secondary generation mechanism. ${ }^{49}$ This is the process in which existing runaway electrons kick thermal electrons into the runaway regime by close Coulomb collisions. This mechanism is predicted theoretically and is thought to dominate the runaway production during disruptions in large tokamaks..$^{50}$ Disruption-generated runaway electrons in TEXTOR have also been identified with this synchrotron radiation diagnostic. ${ }^{51}$

2. In discharges with MHD activity, observations have been made of a fast loss of runaway electrons in the stochastic region outside magnetic islands, whereas within the islands the runaway electrons are almost perfectly confined. These snakelike structures of the runaway beam have been observed at $q=1$ and $q=2$ (Ref. 52).

3. Relativistic runaway electrons can be used to probe the scale size of magnetic turbulence in the core of the plasma. These experiments are done in low-density discharges and heated additionally by NBI. A decay of the runaway population after switch-on of the beam indicated the loss of these electrons because of increased magnetic turbulence. However, a noticeable delay (up to $1 \mathrm{~s}$ ) in the response of the synchrotron signals showed that the loss of runaway electrons occurred at energies well below the energy range of the diagnostic $(20$ to $30 \mathrm{MeV})$. The critical energy for loss of runaway electrons depends on heating power and is related to the scale size of the magnetic turbulence. From these observations it was derived that the radial correlation length of magnetic turbulence in the core of the plasma is $0.2 \mathrm{~cm}$ in ohmic discharges, increasing to several centimeters with 0.6-MW heating. ${ }^{53}$

\section{VI.F. Magnetic Diagnostics}

TEXTOR is equipped with magnetic diagnostics for the determination and real time control of various important plasma parameters including toroidal magnetic field, plasma current, and plasma position.

The plasma current is measured by a Rogowski coil, which surrounds the plasma and is therefore sensitive to the poloidal magnetic field. The position of the plasma is determined from several pickup coils, which sense the changes and the asymmetries in the poloidal field in various directions. The magnetic fields are obtained by integration of the coil signals. Both the Rogowski coil and the pickup coils are part of feedback loops to control the plasma and to keep the current and the vertical and the horizontal positions at their preprogrammed values by applying suitable magnetic correction fields. Another magnetic system is the voltage loop to measure the applied voltage, which is necessary to drive the plasma current and to overcome the resistive losses. This signal is derived from loops parallel to the plasma column and is directly used without integration.

An elegant, but challenging, diagnostic is the measurement of the plasma energy content via the diamagnetism. The diamagnetism of the plasma is proportional to the plasma energy and reduces the toroidal field slightly: Typically, the field is decreased by about $10^{-4}$ of the applied toroidal field. For this measurement, two magnetic coils are used: One of them encloses the plasma and measures the toroidal field that is reduced slightly by the plasma and another coil, which does not enclose the plasma. The fluxes of the two loops are subtracted before integration. This "compensated diamagnetic loop" is capable of measuring very small changes in the flux due to the diamagnetism. Of course, it is also very sensitive to 


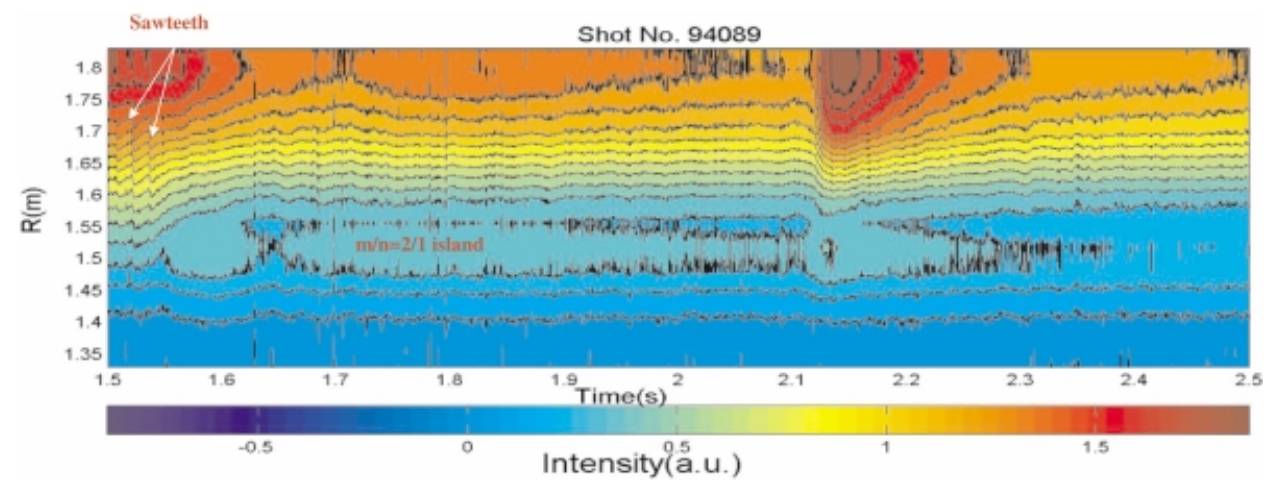

Fig. 21. Contour plot of X-ray intensities taken with one of the vertical cameras (\#v2) of the X-ray tomography systems in a neutral beam-heated DED plasma. At $t=2.1 \mathrm{~s}$ the radiation increases because of a release of impurities.

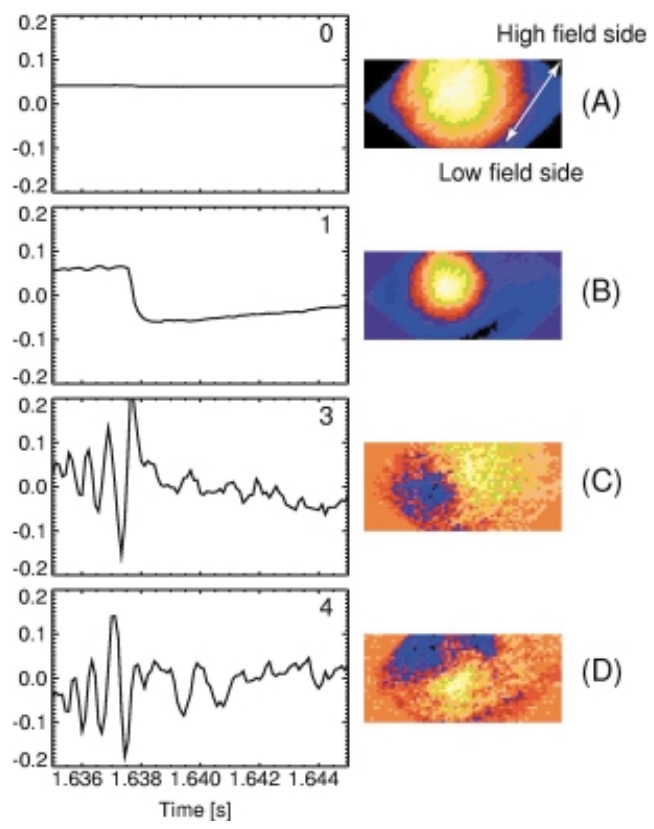

Fig. 22. Singular value decomposed images for the sawtooth instability in TEXTOR taken at $9-\mathrm{kHz}$ framing rate. The time evolution of the four main components (left side) and their spatial pattern (right side) are plotted.

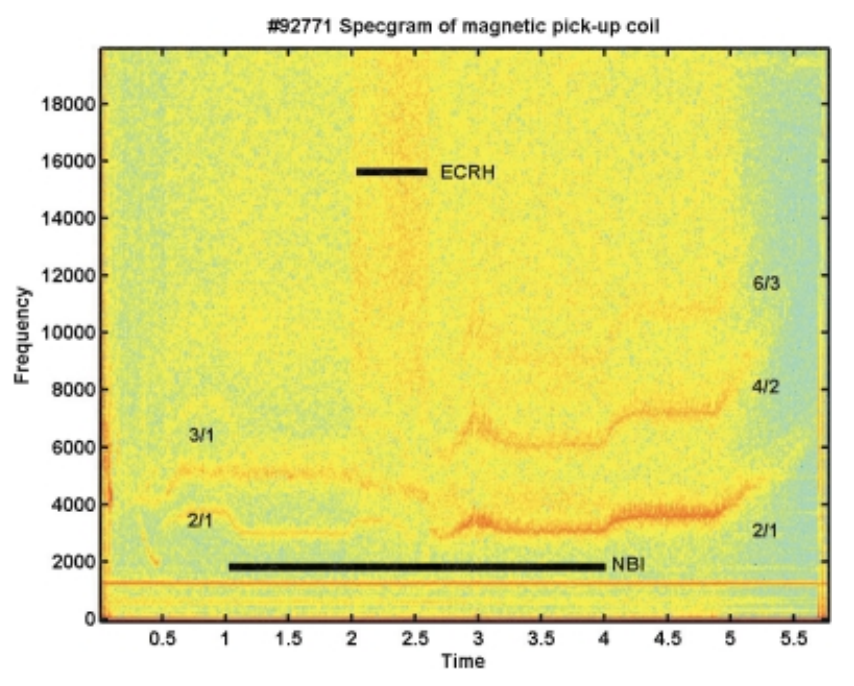

Fig. 25. Spectrogram of a pickup coil during a TEXTOR shot with NBI and ECRH heating.

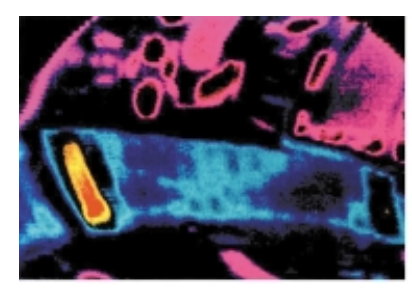

(a)

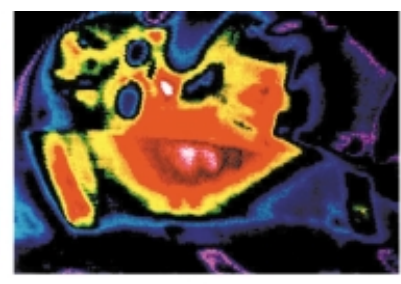

(b)

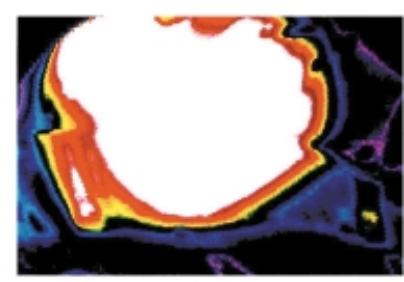

(c)

Fig. 23. Tangential view of a low-density TEXTOR-94 discharge with a thermographic camera looking in the direction of electron approach. In frame A recorded at $t=0.5 \mathrm{~s}$, no synchrotron radiation is observable, and only the wall structure can be recognized. In frame $\mathrm{B}$, recorded at $t=1.5 \mathrm{~s}$, the synchrotron radiation starts to develop, and in frame $\mathrm{C}$ the full extent of the spot is visible from which the size of the runaway beam can be determined. 
misalignments and changes in the area of the coils because of temperature variations, as well as to the rise time of the two coils, due to different magnetic shielding by the surrounding vacuum vessel. On TEXTOR, the signal of the compensated magnetic loop is used to measure the stored energy in the plasma; to measure the ratio $\beta$ between the plasma pressure and the pressure of the magnetic field; and, as the total heating power is known, to derive the energy confinement time. It has been demonstrated in the RI-mode experiments ${ }^{43}$ that stable plasma operation close to the $\beta$ limit is possible by measuring the plasma energy in real time and controlling the heating power to keep the plasma energy just below the $\beta$ limit.

Besides these magnetic probes, which determine plasma properties on timescales from milliseconds to several seconds, fast probes are used to determine magnetic perturbations in the plasma. These perturbations are produced either by the external fields of the DED or as the result of self-organization of the internal magnetic field in the plasma. The magnetic structures cause small perturbations of the magnetic fields outside the plasma. Frequencies up to several hundreds of kilohertz have been observed. The changes in the magnetic fields must be measured simultaneously at different poloidal and toroidal cross sections in order to determine the toroidal and poloidal mode numbers of the magnetic disturbances. Recently, sets of fast Mirnov coils have been installed on TEXTOR. The coils are made of molybdenum wire, and the windings are isolated after each layer of winding by a high-temperature spray ceramic (Fig. 24). The pickup coils are installed as close to the plasma as possible and can be used with temperatures of up to at least $300^{\circ} \mathrm{C}$. Two different stacks of coils are mounted. Each stack consists of coils measuring the radial and

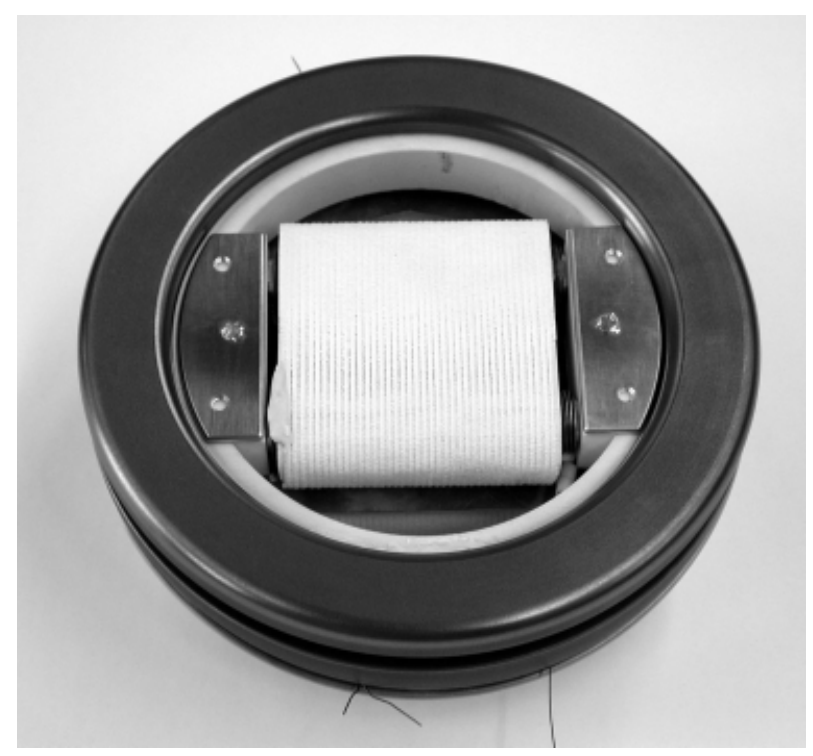

Fig. 24. Pickup coil with a graphite limiter ring. coils measuring the poloidal field component. One coil stack is located in front of the liner-close to the plasmaand the second one is placed behind the liner. An array of eight poloidal coils is fixed at the top of the torus, serving to determine the toroidal mode number, while another array of coils is located in a poloidal cross section in order to analyze the $n$ number of MHD instabilities. The coils facing the plasma are shielded by small carbon limiters. The highest sampling frequency is at present limited to $100 \mathrm{kHz}$ but will be increased in the future. Most coils are routinely sampled at a frequency of $25 \mathrm{kHz}$.

Figure 25 shows the effect of different heating methods on the behavior of MHD instabilities. Low-power NBI coinjection reduces the frequency of the $2 / 1$ mode. The slowing down is in good agreement with a reversal of the plasma rotation that has been observed in the past with NBI coinjection. The spectrogram shows a broadband turbulent feature during the phase with ECRH heating.

The fast Mirnov coils complement the ability of the core diagnostics like ECE, interferometry, and SXR cameras to measure instabilities like sawteeth and tearing modes. Ongoing research is the analysis of the MHD characteristics of the plasma induced by external DED perturbation fields, as well as detailed studies of the penetration and amplification of error fields.

\section{CONCLUSION}

To aid the new physics programs on TEXTOR, described in several other papers in this special issue, it was necessary to upgrade various existing plasma core diagnostics and additionally to install many new and advanced core diagnostics systems. Most of these upgrades and new installations took place in very recent years. Many systems described in this paper are still in the installation and/or commissioning phase. Some of the results shown in this paper are more or less the very first ones, demonstrating that the diagnostic is in principle working (e.g., the results shown for TS and MSE). Nevertheless, some further commissioning is still needed to bring the diagnostics into routine operation. Some other diagnostics are still in the installation phase (e.g., CTS, ultra-SXR tomography), and first results with these are expected early 2005.

Although many of the diagnostics described in this paper are also used, in one or the other form, on other fusion devices, TEXTOR diagnosticians, in collaboration with scientists from several other institutes (as is evidenced by the author list) have in general tried to introduce new technologies or approaches (e.g., multipulse operation of TS, imaging techniques applied to microwave diagnostics, multilayer mirrors used in tomography systems, tangential view for X-ray diagnostics, full spectra-polarimetry for MSE). This is done to 
further enhance the diagnostic capabilities (better resolution, higher accuracy, more channels/chords), with the ultimate aim to better understand the detailed plasma physics processes and contribute to the physics programs that have been presented in other papers in this special issue.

\section{ACKNOWLEDGMENTS}

This work, supported by the European Communities under the contracts of association between EURATOM/FOM and EURATOM/FZJ, was carried out within the framework of the European Fusion Development Agreement, with financial support from the Nederlandse Organisatie voor Wetenschappelijk Onderzoek, Forschungszentrum Jülich $\mathrm{GmbH}$, and EURATOM. The views and opinions expressed herein do not necessarily reflect those of the European Commission.

\section{REFERENCES}

1. O. NEUBAUER et al., "Design Features of the Tokamak TEXTOR," Fusion Sci. Technol., 47, 76 (2005).

2. V. PHILIPPS, "Wall Conditioning on TEXTOR," Fusion Sci. Technol., 47, 119 (2005). B. SCHWEER et al., "Limiter Lock Systems at TEXTOR: Flexible Tools for Plasma-Wall Investigation," Fusion Sci. Technol., 47, 138 (2005).

3. A. J. H. DONNÉ et al., "New Diagnostics for Physics Studies on TEXTOR-94," Rev. Sci. Instrum., 72, 1046 (2001).

4. D. VÉRON, "Sub-Millimeter Interferometry of High-Density Plasmas," Infrared and Millimeter Waves, Vol. 2, p. 69, K. J. BUTTON, Ed., Academic Press, New York (1979).

5. F. DE MARCO and S. E. SEGRE, "The Polarization of an E.M. Wave Propagating in a Plasma with Magnetic Shear. The Measurement of Poloidal Magnetic Field in a Tokamak," Plasma Phys., 14, 245 (1972).

6. H. R. KOSLOWSKI, "A Real-Time Multiradian Phase Detector for Interferometry Based on a Digital Phase Locked Loop Circuit," Meas. Sci. Technol., 5, 307 (1994).

7. H. R. KOSLOWSKI and H. SOLTWISCH, "Electron Density and q Profile Measurements with Far-IR Interferometer-Polarimeter on the TEXTOR Tokamak," Fusion Eng. Des., 34-35, 143 (1997).

8. C. J. BARTH et al., "High-resolution Multiposition Thomson Scattering on TEXTOR," Rev. Sci. Instrum., 72, 1138 (2001).

9. P. C. DE VRIES et al., "Density Profile Peaking Inside $m / n=2 / 1$ Magnetic Islands in TEXTOR-94," Nucl. Fusion, 37, 1641 (1997).

10. M. YU. KANTOR et al., "Test of a Periodic Multipass-Intracavity Laser System for the TEXTOR Multiposition Thomson Scattering System,” Rev. Sci. Instrum., 72, 1159 (2001).

11. H. J. VAN DER MEIDEN et al., "10 kHz Repetitive HighResolution TV Thomson Scattering on TEXTOR," Rev. Sci. Instrum., 75, 3849 (2004).

12. M. JADOUL et al., "Time-Dependent Analysis of Spatially Resolved Fluctuations Observed by Microwave Scattering on TEXTOR," Plasma Phys. Control. Fusion, 40, 381 (1998).

13. G. WAIDMANN et al., "Multichannel Electron Cyclotron Emission Radiometry for Magnetohydrodynamic Mode Studies in TEXTOR,” Rev. Sci. Instrum., 68, 492 (1997).
14. A. KRÄMER-FLECKEN, G. WAIDMANN, and P. C. DE VRIES, "Measurement of 3rd Harmonic of ECE-Radiation at TEXTOR-94," Proc. 10th Joint Workshop Electron Cyclotron Emission and Electron Cyclotron Heating, Ameland, The Netherlands, 1997, p. 209, A. J. H. DONNÉ and A. G. A. VERHOEVEN, Eds., World Scientific (1997).

15. V. S. UDINTSEV et al., "New ECE Diagnostics for the TEXTOR-94 Tokamak," Rev. Sci. Instrum., 72, 359 (2001).

16. V. S. UDINTSEV, "Electron Temperature Dynamics of TEXTOR Plasmas," Thesis, University of Utrecht (2003).

17. J. C. VAN GORKOM et al., "The Ten-Channel Pulsed Radar Reflectometer at the TEXTOR Tokamak," Rev. Sci. Instrum., 72, 336 (2001).

18. V. S. UDINTSEV et al., "Studies of Plasma Transport Properties in Presence of Large and Small MHD Modes at TEXTOR," Nucl. Fusion, 43, 1424 (2003).

19. H. PARK et al., "Recent Advancements in Microwave Imaging Plasma Diagnostics,” Rev. Sci. Instrum., 74, 4239 (2003).

20. E. MAZZUCATO, "Microwave Reflectometry for Magnetically Confined Plasmas," Rev. Sci. Instrum., 69, 2201 (1998).

21. B. H. DENG et al., "ECE Imaging of Plasma $T_{e}$ Profiles and Fluctuations," Fusion Eng. Des., 53, 77 (2001).

22. C. W. DOMIER et al., "3-D Fluctuation Imaging Diagnostic for TEXTOR,” Rev. Sci. Instrum., 72, 363 (2001).

23. T. MUNSAT et al., "Laboratory Characterization of an Imaging Reflectometer System," Plasma Phys. Control. Fusion, 45, 469 (2003).

24. H. BINDSLEV et al., "Fast Ion Dynamics Measured by Collective Thomson Scattering of mm Waves," Proc. 10th Int. Symp. Laser-Aided Plasma Diagnostics, Fukuoka, Japan, 2001, p. 97, K. MURAOKA, Ed., Kyushu University (2001).

25. S. MICHELSEN et al., "Fast Ion Millimeter Wave CTS Diagnostics on TEXTOR and ASDEX Upgrade," Rev. Sci. Instrum., 75, 3634 (2004).

26. A. J. H. DONNÉ, E. P BARBIAN, and H. W. VAN DER VEN, "On the Application of the Rutherford-Scattering Diagnostic to Ion Temperature Measurements," J. Appl. Phys., 62, 3130 (1987).

27. A. A. E. VAN BLOKLAND et al., "Ion Temperature Measurements in Tokamak Plasmas by Rutherford Scattering," Rev. Sci. Instrum., 63, 3359 (1992).

28. A. A. E. VAN BLOKLAND, T. W. M. GRIMBERGEN, and H. W. VAN DER VEN, "A Mass-Selective Neutral Particle Energy Analyzer Providing a Background Rejection Capability," Rev. Sci. Instrum., 63, 1978 (1992).

29. H. F. TAMMEN et al., "Rutherford Scattering at TEXTOR," Rev. Sci. Instrum., 63, 4583 (1992).

30. H. F. TAMMEN et al., "The Investigation of Ion Sawtooth Activity by Rutherford Scattering," Rev. Sci. Instrum., 66, 327 (1995).

31. H. F. TAMMEN et al., "First Measurements of the Toroidal Rotation of the Bulk Ions at TEXTOR by Rutherford Scattering," Phys. Rev. Lett., 72, 356 (1994).

32. G. BERTSCHINGER, D. REITER, and L. KÖNEN, "Determination of the Flux of Thermal Neutral Particles from the Wall of a Tokamak by Pulsed Gas Injection," Proc. 12th European Conf. Controlled Fusion and Plasma Physics, Budapest, Hungary, 1985, p. 251, European Physics Society, Geneva.

33. E. BUSCHE, H. EURINGER, and R. JASPERS, "Measurement of Deuterium Ion Temperature Profiles at TEXTOR-94," Plasma Phys. Contr. Fusion, 39, 1327 (1997).

34. A. KRETER et al., "Investigation of Ion Transport in Ohmic Discharges by Charge-Exchange Recombination Spectroscopy with a 
Diagnostic Hydrogen Beam in TEXTOR-94," Proc. 27th European Physical Society Conf. Controlled Fusion and Plasma Physics, Budapest, Hungary, June 12-16, 2000, Europhysics Conference Abstracts, Vol. 24B, p. 1232 (2000).

35. R. JASPERS et al., "Spectra-Polarimetry of the Motional Stark Effect at TEXTOR-94," Rev. Sci. Instrum., 72, 1018 (2001).

36. K. JAKUBOWSKA et al., "The Motional Stark Effect Diagnostic in the Tokamak TEXTOR," Rev. Sci. Instrum., 75, 3475 (2004).

37. R. FONCK, A. T. RAMSEY, and R. V. YELLE, "Multichannel Grazing-Incidence Spectrometer for Plasma Impurity Diagnosis: SPRED," Appl. Opt., 21, 2115 (1982).

38. W. BIEL et al., "Detector System with High Time Resolution for the Continuous Measurement of Spectra in the Vacuum Ultraviolet Wavelength Range," Rev. Sci. Instrum., 75, 471 (2004).

39. W. BIEL and TEXTOR TEAM, "Vacuum Ultraviolet Spectroscopy at TEXTOR,” Fusion Sci. Technol., 47, 246 (2005).

40. L. SHMAENOK et al., "A Novel Instrumentation for Spectrally Resolved Soft X-Ray Plasma Tomography: Development and Pilot Results on TEXTOR," Rev. Sci. Instrum., 72, 1411 (2001).

41. M. DE BOCK et al., "Measuring 1D and 2D Impurity Density Profiles on TEXTOR Using Combined CXRS, BES and Ultra Soft X-Ray Tomography," Rev. Sci. Instrum., 75, 4155 (2004).

42. G. BERTSCHINGER et al., "X-Ray Spectroscopy at TEXTOR," Fusion Sci. Technol., 47, 253 (2005).

43. B. UNTERBERG et al., "The Radiative Improved Mode in TEXTOR: Power Exhaust and Improved Confinement at High Density," Fusion Sci. Technol., 47, 187 (2005).
44. J. RAPP, "Ortsaufgelöste Messung der Strahlungsleistung des TEXTOR-Plasmas in einem poloidalen Querschnitt," PhD Thesis, University of Wuppertal (1996); see also Rep. Forschungszentrum Jülich Jül-3172 (1996) (in German)

45. S. OHDACHI et al., "High-Speed Tangentially Viewing Soft X-Ray Camera to Study Magnetohydrodynamic Fluctuations in Toroidally Confined Plasmas," Rev. Sci. Instrum., 74, 2136 (2003).

46. C. NARDONE, "Multichannel Fluctuation Data Analysis by the Singular Value Decomposition Method. Application to MHD Modes in JET," Plasma Phys. Control. Fusion, 34, 1447 (1992).

47. K. H. FINKEN et al., "Observation of Infrared Synchrotron Radiation from Tokamak Runaway Electrons in TEXTOR," Nucl. Fusion, 30, 859 (1990).

48. R. JASPERS et al., "A Synchrotron Radiation Diagnostic to Observe Relativistic Runaway Electrons in a Tokamak Plasma," Rev. Sci. Instrum., 72, 466 (2001).

49. R. JASPERS et al., "Experimental Investigation of Runaway Electron Generation in TEXTOR,” Nucl. Fusion, 33, 1775 (1993).

50. S. PUTVINSKI et al., "Halo Current, Runaway Electrons and Disruption Mitigation in ITER," Plasma Phys. Control. Fusion, 39, B157 (1997).

51. R. JASPERS et al., "Disruption Generated Runaway Electrons in TEXTOR and ITER,” Nucl. Fusion, 36, 367 (1996).

52. R. JASPERS et al., "Islands of Runaway Electrons in the TEXTOR Tokamak and Relation to Transport in a Stochastic Field," Phys. Rev. Lett., 72, 4093 (1994); see also I. ENTROP et al., "Runaway Snakes in TEXTOR-94," Plasma Phys. Control. Fusion, 41, 377 (1999).

53. I. ENTROP et al., "Scale Size of Magnetic Turbulence in Tokamaks Probed with 30-MeV Electrons," Phys. Rev. Lett., 84, 3606 (2000). 